\title{
A higher order numerical method for time fractional partial differential equations with nonsmooth data
}

\author{
Yanyuan Xing ${ }^{\mathrm{a}}$, Yubin Yan*,b \\ ${ }^{a}$ Department of Mathematics, Lvliang University, Lishi, P.R.China, 033000 \\ ${ }^{b}$ Department of Mathematics, University of Chester, CH1 $4 \mathrm{BJ}$
}

\begin{abstract}
Gao et al. [12] (2014) introduced a numerical scheme to approximate the Caputo fractional derivative with the convergence rate $O\left(k^{3-\alpha}\right), 0<\alpha<1$ by directly approximating the integer-order derivative with some finite difference quotients in the definition of the Caputo fractional derivative, see also $\mathrm{Lv}$ and $\mathrm{Xu}$ [22] (2016), where $k$ is the time step size. Under the assumption that the solution of the time fractional partial differential equation is sufficiently smooth, Lv and Xu [22] (2016) proved by using energy method that the corresponding numerical method for solving time fractional partial differential equation has the convergence rate $O\left(k^{3-\alpha}\right), 0<\alpha<1$ uniformly with respect to the time variable $t$. However, in general the solution of the time fractional partial differential equation has low regularity and in this case the numerical method fails to have the convergence rate $O\left(k^{3-\alpha}\right), 0<\alpha<1$ uniformly with respect to the time variable $t$. In this paper, we first obtain a similar approximation scheme to the Riemann-Liouville fractional derivative with the convergence rate $O\left(k^{3-\alpha}\right), 0<\alpha<1$ as in Gao et al. [12] (2014) by approximating the Hadamard finitepart integral with the piecewise quadratic interpolation polynomials. Based on this scheme, we introduce a time discretization scheme to approximate the time fractional partial differential equation and show by using Laplace transform methods that the time discretization scheme has the convergence rate $O\left(k^{3-\alpha}\right), 0<\alpha<1$ for any fixed $t_{n}>0$ for smooth and nonsmooth data in both homogeneous and inhomogeneous cases. Numerical examples are given to show that the theoretical results are consistent with the numerical results.
\end{abstract}

Key words:

Time fractional partial differential equation, error estimates, Laplace transform, Caputo fractional derivative AMS Subject Classification: 65M12; 65M06; 65M70; 35 S10

\section{Introduction}

Consider the following time fractional partial differential equation, with $0<\alpha<1$,

$$
{ }_{0}^{C} D_{t}^{\alpha} u(t)+A u(t)=f(t), \quad \text { for } 0<t \leq T, \quad \text { with } u(0)=u_{0},
$$

where ${ }_{0}^{C} D_{t}^{\alpha} u(t)$ denotes the Caputo fractional derivative defined by

$$
{ }_{0}^{C} D_{t}^{\alpha} u(t)=\frac{1}{\Gamma(1-\alpha)} \int_{0}^{t}(t-s)^{-\alpha} u^{\prime}(s) d s,
$$

and $u^{\prime}(s)=\partial u / \partial s$. Here $A$ is a selfadjoint positive definite second order elliptic partial differential operator in a bounded regular domain $\Omega \subset \mathbb{R}^{d}, d=1,2,3$, with $\mathcal{D}(A)=H_{0}^{1}(\Omega) \cap H^{2}(\Omega)$ and $u_{0} \in H=L_{2}(\Omega)$ is the initial value, where $L_{2}(\Omega), H_{0}^{1}(\Omega), H^{2}(\Omega)$ denote the standard Sobolev spaces.

\footnotetext{
*Corresponding author

Email addresses: 1lxysxxxyy@163.com ( Yanyuan Xing), y.yan@chester.ac.uk (Yubin Yan) 
Our analysis will use Laplace transforms. The assumption that $A$ is positive definite implies that $A$ generates an analytic semigroup, so that for some $\pi / 2<\theta_{0}<\pi$ we have the resolvent estimate, see Lubich et al. [23], Thomée [40]

$$
\left\|(z I+A)^{-1}\right\| \leq C|z|^{-1} \text { for } z \in \Sigma_{\theta_{0}}=\left\{z \neq 0:|\arg z|<\theta_{0}\right\} .
$$

In our analysis, we will choose $\theta>\pi / 2$ close to $\pi / 2$ such that $\theta<\theta_{0}$. Hence $z^{\alpha} \in \Sigma_{\theta_{0}}$ for any $z \in \Sigma_{\theta}$ since $0<\alpha<1$ implies that $\arg \left(z^{\alpha}\right)=\alpha \theta<\theta<\theta_{0}$. Hence there exists a constant $C$ which depends only on $\theta$ and $\alpha$ such that, see Jin et al. [16, (2.3)],

$$
\left\|\left(z^{\alpha} I+A\right)^{-1}\right\| \leq C|z|^{-\alpha}, \quad \forall z \in \Sigma_{\theta}=\{z \neq 0:|\arg \mathrm{z}|<\theta\} .
$$

Further we will choose $\theta>\pi / 2$ close to $\pi / 2$ such that $z_{k}^{\alpha} \in \Sigma_{\theta_{0}}$ for $z \in \Gamma$ (see Lemma 2.9 below) which implies that $\left(z_{k}^{\alpha} I+A\right)^{-1}$ exists where $z_{k}$ is defined in $(25)$ and $\Gamma=\Gamma_{\theta}=\{z:|\arg z|=\theta\}$.

Many application problems can be modeled by (1), for example, thermal diffusion in media with fractional geometry [29], highly heterogeneous aquifer [1], underground environmental problems [14], random walks [13], etc.

Under the assumptions that the solution $u \in C^{m}[0, T], m \in \mathbb{N}, m \geq 2$ in time, the error estimates of some numerical methods are analyzed in the literature, see, e.g., [19], [21], [12], [35], [20], [38], [43], [11], [10], [37], etc.

However, in view of the smoothing property of the time fractional partial differential equation, the regularity $u \in C^{m}[0, T], m \geq 2$ in time is restrictive, since it does not hold true even for homogeneous problem with the smooth initial data, see, e.g., Sakamoto and Yamamoto [32] and Jin et al. [16]. Jin et al. [16] approximated the Caputo fractional derivative by $L 1$ scheme and established an $O(k)$ convergence rate for both smooth and nonsmooth initial data. Recently, Yan et al. [36] introduced an improved L1 scheme for solving (1) and established an $O\left(k^{2-\alpha}\right)$ convergence rate for both smooth and nonsmooth initial data. Jin et al. [18] introduced the Crank-Nicolson scheme for solving (1) and a second-order accuracy in time was established for both smooth and nonsmooth initial data, see also [24], [27], etc.

There has been much recent interest in developing higher order numerical methods for solving (1), especially the spectral methods, [3], [4], [41], [42], convolution quadrature correction methods, [44], [43], [5], the discontinuous Galerkin method [6], [25], [26], [28], nonuniform meshes, [33], [34], [31], [39],

In this paper, we first introduce a higher order scheme to approximate the Riemann-Liouville fractional derivative with order $O\left(k^{3-\alpha}\right), 0<\alpha<1$, which is similar to the approximate methods introduced in [12] and [22]. The scheme is obtained by approximating the Hadamard finite-part integral with piecewise quadratic polynomials [7] [8]. Based on this higher order scheme, we introduce a time discretization method for solving (1) and the convergence rate $O\left(k^{3-\alpha}\right)$ of the numerical method is proved for smooth and nonsmooth initial data in both homogeneous and inhomogeneous cases.

The paper is organized as follows. In Section 2, we consider the error estimates of the time discretization scheme for solving (1) for smooth and nonsmooth initial data in the homogeneous case. In Section 3 , we consider the error estimates of the numerical methods for solving (1) for smooth and nonsmooth initial data in the inhomogeneous case. Finally in Section 4, we give some numerical examples to show that the numerical results are consistent with the theoretical results.

By $C$ we denote a positive constant independent of the functions and parameters concerned, but not necessarily the same at different occurrences. By $c$ we denote a particular positive constant independent of the functions and parameters concerned.

\section{The homogeneous problem}

In this section, we will consider the time discretization scheme for (1) in the homogeneous case. Let $V(t)=u(t)-u_{0}$, then $(1)$ is equivalent to

$$
{ }_{0}^{R} D_{t}^{\alpha} V(t)+A V(t)=-A u_{0}, \quad 0<t \leq T, \quad \text { with } V(0)=0,
$$


where we used the fact ${ }_{0}^{R} D_{t}^{\alpha}(u(t)-u(0))={ }_{0}^{C} D_{t}^{\alpha} u(t)$ [7]. Here ${ }_{0}^{R} D_{t}^{\alpha} u(t)$ denotes the Riemann-Liouville fractional derivative defined by, with $0<\alpha<1$,

$$
{ }_{0}^{R} D_{t}^{\alpha} u(t)=\frac{1}{\Gamma(1-\alpha)} \frac{d}{d t} \int_{0}^{t}(t-s)^{-\alpha} u(s) d s .
$$

Let $0=t_{0}<t_{1}<\cdots<t_{N}=T$ be a partition of $[0, T]$ with the time step size $k$. Diethelm [7] introduced the following approximation scheme to approximate the Riemann-Liouville fractional derivative, with some sufficiently smooth function $u$ and $0<\alpha<1$,

$$
{ }_{0}^{R} D_{t}^{\alpha} u\left(t_{n}\right)=k^{-\alpha} \sum_{j=0}^{n} w_{j, n} u\left(t_{n-j}\right)+O\left(k^{2-\alpha}\right),
$$

where

$$
\Gamma(2-\alpha) w_{j, n}=\left\{\begin{array}{l}
1, \quad j=0, \\
-2 j^{1-\alpha}+(j-1)^{1-\alpha}+(j+1)^{1-\alpha}, \quad j=1,2, \ldots, n-1, \\
(j-1)^{1-\alpha}-j^{1-\alpha}, \quad j=n .
\end{array}\right.
$$

We remark that the approximation scheme (5) to the Riemann-Liouville fractional derivative can also be obtained by first approximating the Caputo fractional derivative with the L1 scheme introduced in, e.g, [21] [16] and then applying the relation between the Caputo and Riemann-Liouville fractional derivatives.

Define the following time discretization scheme for solving (4), with $V^{j} \approx V\left(t_{j}\right), j=0,1,2, \ldots, n$, $n=1,2, \ldots, N$,

$$
k^{-\alpha} \sum_{j=0}^{n} w_{n-j, n} V^{j}+A V^{n}=-A u_{0}, \quad V^{0}=0,
$$

or

$$
k^{-\alpha} \sum_{j=1}^{n} w_{n-j, n} V^{j}+A V^{n}=-A u_{0} .
$$

Jin et al. [16, Theorem 3.16] proved the following theorem for the nonsmooth initial data $u_{0}$.

Theorem 2.1. Let $V\left(t_{n}\right)$ and $V^{n}$ be the solutions of (4) and (7), respectively. Let $u_{0} \in L_{2}(\Omega)$. Then we have

$$
\left\|V^{n}-V\left(t_{n}\right)\right\| \leq C k t_{n}^{-1}\left\|u_{0}\right\| .
$$

In order to achieve higher accuracy, by modifying the approximation scheme (6) at the first time step $n=1$, Yan et al. [36] introduced the following modified L1 scheme: with $c_{0}=1 / 2$,

$$
\begin{aligned}
& k^{-\alpha} \sum_{j=1}^{n} w_{n-j, n} V^{j}+A V^{n}=\left(-A u_{0}\right)\left(1+c_{0}\right), \quad n=1, \\
& k^{-\alpha} \sum_{j=1}^{n} w_{n-j, n} V^{j}+A V^{n}=-A u_{0}, \quad n=2,3, \ldots N, \\
& V^{0}=0,
\end{aligned}
$$

and show the following result in [36].

Theorem 2.2. Let $c_{0}=1 / 2$ and let $V\left(t_{n}\right)$ and $V^{n}$ be the solutions of (4) and (8)-(10), respectively, Let $u_{0} \in L_{2}(\Omega)$. Then we have

$$
\left\|V^{n}-V\left(t_{n}\right)\right\| \leq C k^{2-\alpha} t_{n}^{\alpha-2}\left\|u_{0}\right\| .
$$


In this section, we will introduce the following time discretization scheme to approximate (4), with $a_{1}=11 / 12, a_{2}=-5 / 12$,

$$
\begin{aligned}
& k^{-\alpha} \sum_{j=1}^{n} w_{n-j} V^{j}+A V^{n}=\left(-A u_{0}\right)\left(1+a_{1}\right), \quad n=1, \\
& k^{-\alpha} \sum_{j=1}^{n} w_{n-j} V^{j}+A V^{n}=\left(-A u_{0}\right)\left(1+a_{2}\right), \quad n=2, \\
& k^{-\alpha} \sum_{j=1}^{n} w_{n-j} V^{j}+A V^{n}=-A u_{0}, \quad n=3,4, \ldots N, \\
& V^{0}=0,
\end{aligned}
$$

where $w_{j}, j=0,1,2, \ldots$ are determined by (16) below.

Remark 2.1. The parameter $c_{0}$ in (8)-(10) and the parameters $a_{1}$ and $a_{2}$ in (11)-(12) are very important. For example, the accuracy of the numerical method (11)-(14) is sensitive to the values $V^{1}$ and $V^{2}$. One must use (11) and (12) to calculate the starting values $V^{1}$ and $V^{2}$. In other words, one can not get the required accuracy $O\left(k^{3-\alpha}\right)$ of the numerical method (11)-(14) at time $T$ if one use other methods to calculate the starting values $V^{1}, V^{2}$. In numerical example 4.3 in Section 4 , we observe that the numerical method (11)-(14) fails to get the required accuracy even we use the exact starting values $V^{1}, V^{2}$.

Theorem 2.3. Let $a_{1}=11 / 12, a_{2}=-5 / 12$ and let $V\left(t_{n}\right)$ and $V^{n}$ be the solutions of (4) and (11)-(14), respectively. Let $u_{0} \in L_{2}(\Omega)$. Then there exists a positive constant $C$ such that

$$
\left\|V^{n}-V\left(t_{n}\right)\right\| \leq C k^{3-\alpha} t_{n}^{\alpha-3}\left\|u_{0}\right\| .
$$

Let us first introduce the weights $w_{j}, j=0,1,2, \ldots$ in (11)-(14). To do this, we need to introduce a higher order numerical method to approximate Riemann-Liouville fractional derivative. For simplicity of the notations of the weights in the approximation scheme to the Riemann-Liouville fractional derivative in Lemma 2.4 below, we will only consider the weights with $n \geq 5$. Similarly one may consider the weights with $n \leq 5$ separately. For our numerical methods (11)-(14), the approximate solutions $V^{j}, j=1,2, \ldots, n$ with $n \geq 1$ are determined only by the weights $w_{j}, j=0,1,2, \ldots, n-1$ defined by (16). In other words, it is not necessary to consider the starting values approximations $V^{1}, V^{2}, V^{3}, V^{4}$ by using other ways when we calculate the solutions $V^{n}$ with $n \geq 5$. We may apply the same method (11)-(14) to obtain all $V^{n}$ with $n \geq 1$.

Lemma 2.4. Let $0<\alpha<1$ and assume that $u \in C^{3}[0, T]$. Let $n \geq 5$, then we have

$$
{ }_{0}^{R} D_{t}^{\alpha} u\left(t_{n}\right)=k^{-\alpha} \sum_{j=0}^{n} w_{j, n} u\left(t_{n-j}\right)+O\left(k^{3-\alpha}\right),
$$

where

$$
\Gamma(3-\alpha) w_{j, n}=\left\{\begin{array}{l}
\left(1+\frac{\alpha}{2}\right) 2^{1-\alpha}, \quad j=0, \\
\left(2+\frac{\alpha}{2}\right) 3^{1-\alpha}-\left(3+\frac{3}{2} \alpha\right) 2^{1-\alpha}, \quad j=1, \\
\left(3+\frac{\alpha}{2}\right) 4^{1-\alpha}-\left(6+\frac{3}{2} \alpha\right) 3^{1-\alpha}+\left(3+\frac{3}{2} \alpha\right) 2^{1-\alpha}, \quad j=2,
\end{array}\right.
$$

and, with $j=3,4, \ldots, n-2$,

$$
\begin{aligned}
\Gamma(3-\alpha) w_{j, n} & =(j+2)^{2-\alpha}-\left(1-\frac{\alpha}{2}\right)(j+2)^{1-\alpha}-3(j+1)^{2-\alpha} \\
& +\left(3-\frac{3}{2} \alpha\right)(j+1)^{1-\alpha}+3 j^{2-\alpha}-\left(3-\frac{3 \alpha}{2}\right) j^{1-\alpha}-(j-1)^{2-\alpha} \\
& +\left(1-\frac{\alpha}{2}\right)(j-1)^{1-\alpha}, \quad j=3,4, \ldots n-2
\end{aligned}
$$


and

$$
\begin{aligned}
& \Gamma(3-\alpha) w_{n-1, n}=(4-2 n-2 \alpha) n^{1-\alpha}+\left(3 n-6+\frac{3 \alpha}{2}\right)(n-1)^{1-\alpha}-\left(n+\frac{\alpha}{2}-3\right)(n-2)^{1-\alpha}, \\
& \Gamma(3-\alpha) w_{n, n}=\left(n-3+\frac{3 \alpha}{2}\right) n^{1-\alpha}+(1-\alpha)(2-\alpha) n^{-\alpha}-\left(n-2+\frac{\alpha}{2}\right)(n-1)^{1-\alpha} .
\end{aligned}
$$

Proof: See the Appendix.

We remark that for any fixed $n \geq 5, w_{j, n}, j=0,1, \ldots, n-2$ are independent on $n$ and only $w_{n-1, n}$ and $w_{n, n}$ depend on $n$. For example, we have $w_{0, n}=\left(1+\frac{\alpha}{2}\right) 2^{1-\alpha}$ for any $n \geq 5, w_{1, n}=\left(2+\frac{\alpha}{2}\right) 3^{1-\alpha}-\left(3+\frac{3}{2} \alpha\right) 2^{1-\alpha}$ for any $n \geq 5, \ldots$ Based on this observation, we may define $w_{j}, j=0,1,2, \ldots, n-1$ which we need in the numerical scheme (11)-(14) as follows. For $j=0,1,2$, we define $w_{0}=w_{0, n}, w_{1}=w_{1, n}, w_{2}=w_{2, n}$ with $n \geq 5$. For $w_{j}, j=3,4, \ldots, n-2$, we define $w_{j}=w_{j, n}, j=3,4, \ldots, n-2$, where

$$
\begin{aligned}
\Gamma(3-\alpha) w_{j, n}= & \frac{1}{2} E_{j+2}-F_{j+1}+\frac{1}{2} G_{j}=(j+2)^{2-\alpha}-\left(1-\frac{\alpha}{2}\right)(j+2)^{1-\alpha}-3(j+1)^{2-\alpha} \\
& +\left(3-\frac{3}{2} \alpha\right)(j+1)^{1-\alpha}+3 j^{2-\alpha}-\left(3-\frac{3 \alpha}{2}\right) j^{1-\alpha}-(j-1)^{2-\alpha}+\left(1-\frac{\alpha}{2}\right)(j-1)^{1-\alpha} .
\end{aligned}
$$

For $w_{j}, j \geq n-1$, we also define $w_{j}$ by

$$
\begin{aligned}
\Gamma(3-\alpha) w_{j}= & \frac{1}{2} E_{j+2}-F_{j+1}+\frac{1}{2} G_{j}=(j+2)^{2-\alpha}-\left(1-\frac{\alpha}{2}\right)(j+2)^{1-\alpha}-3(j+1)^{2-\alpha} \\
& +\left(3-\frac{3}{2} \alpha\right)(j+1)^{1-\alpha}+3 j^{2-\alpha}-\left(3-\frac{3 \alpha}{2}\right) j^{1-\alpha}-(j-1)^{2-\alpha}+\left(1-\frac{\alpha}{2}\right)(j-1)^{1-\alpha} .
\end{aligned}
$$

Now for any fixed $n \geq 5$, we have defined all $w_{j}, j=0,1,2, \ldots, n-2, n-1, n, \ldots$ We remark that in our numerical method (11)-(14), we only need the weights $w_{j}, j=0,1,2, \ldots, n-1$, where $w_{j}=w_{j, n}, j=$ $0,1,2, \ldots, n-2$, but $w_{n-1} \neq w_{n-1, n}$. We shall see that in the proof of Theorem 2.3 , we need to define all $w_{j}, j=0,1,2, \ldots, n-2, n-1, n, \ldots$ in order to use the discrete Laplace transform. For $w_{j}, j=$ $0,1,2, \ldots, n-2, n-1, n, \ldots$ we introduce the so called discrete Laplace transform, [23]

$$
\widetilde{w}(\zeta):=\sum_{j=0}^{\infty} w_{j} \zeta^{j}
$$

Remark 2.2. In the numerical method (11)-(14), the weights $w_{j}, j=0,1,2, \ldots n-1$ are not completely consistent with the weights $w_{j, n}, j=0,1,2, \ldots, n-1$ defined in (15). We modified the weight $w_{n-1}$ such that $w_{n-1}$ has the same form as $w_{j}, j=3,4, \ldots, n-2$ for $n \geq 5$ and we also define all $w_{j}, j=n, n+1, \ldots$ in (16) since we need to apply the discrete Laplace transform $\sum_{j=0}^{\infty} w_{j} \zeta^{j}$ in our analysis later.

Lemma 2.5. We have the following singularity expansion, with $\zeta=e^{-z k}$,

$$
\sum_{j=0}^{\infty} w_{j} \zeta^{j}=(z k)^{\alpha}+d_{2}(z k)^{3}+d_{3}(z k)^{3+\alpha}+\ldots
$$

for some suitable constants $d_{2}, d_{3}, \ldots$

To prove Lemma 2.5, we need to introduce the polylogarithm function

$$
L i_{p}(z)=\sum_{j=1}^{\infty} \frac{z^{j}}{j^{p}}
$$


The polynomial function $\operatorname{Li}_{p}(z)$ is well defined for $|z|<1$ and $p \in \mathbb{C}$. It can be analytically continued to the split complex plane $\mathbb{C} \backslash[1,+\infty)$; see Flajolet [9] with $z=1$, it recovers the Riemann zata function $\varsigma(p)=L i_{p}(1)$, we also recall an important singular expansion of the function $L i_{p}\left(e^{-z}\right)$ (Flajolet [9], Theorem $1)$.

Lemma 2.6. ( $[16]$, Lemma 3.2) For $p \neq 1,2, \ldots$, the function $L i_{p}\left(e^{-z}\right)$ satisfies the singular expansion

$$
L i_{p}\left(e^{-z}\right) \sim \Gamma(1-p) z^{p-1}+\sum_{l=0}^{\infty}(-1)^{l} \varsigma(p-l) \frac{z^{l}}{l !},
$$

where $\varsigma(z)$ denotes the Riemann zeta function.

Lemma 2.7. ([16], Lemma 3.4) Let $|z| \leq \frac{\pi}{\sin \theta}$ with $\theta \in\left(\frac{\pi}{2}, \frac{5 \pi}{6}\right)$, and $-1<p<0$. Then

$$
L i_{p}\left(e^{-z}\right)=\Gamma(1-p) z^{p-1}+\sum_{l=0}^{\infty}(-1)^{l} \varsigma(p-l) \frac{z^{l}}{l !},
$$

converges absolutely.

Proof of Lemma 2.5: We have, by the definition of $\widetilde{w}(z)$ in (16) with $\zeta=e^{-z k}$,

$$
\begin{aligned}
\widetilde{w}(z)= & \sum_{j=0}^{\infty} w_{j} \zeta^{j}=\frac{1}{\Gamma(3-\alpha)}\left(\zeta^{-2}-3 \zeta^{-1}+3-\zeta\right)\left(\sum_{j=1}^{\infty} j^{2-\alpha} \zeta^{j}\right) \\
& +\frac{1}{\Gamma(3-\alpha)}\left[\left(3-\frac{3 \alpha}{2}\right) \zeta^{-1}-\left(1-\frac{\alpha}{2}\right) \zeta^{-2}-\left(3-\frac{3 \alpha}{2}\right)+\left(1-\frac{\alpha}{2}\right) \zeta\right]\left(\sum_{j=1}^{\infty} j^{1-\alpha} \zeta^{j}\right) \\
& +\frac{1}{\Gamma(3-\alpha)}\left(\frac{\alpha}{2} \zeta^{2}-\frac{3 \alpha}{2} \zeta+\frac{3 \alpha}{2}-\frac{\alpha}{2} \zeta^{-1}\right) \\
= & \frac{1}{\Gamma(3-\alpha)}\left[(z k)^{3}+\frac{1}{2}(z k)^{4}+\frac{1}{4}(z k)^{5}+\ldots\right] L i_{\alpha-2}(\zeta) \\
& +\frac{1}{\Gamma(3-\alpha)}\left[\left(-1+\frac{\alpha}{2}\right)(z k)^{3}+\left(-\frac{1}{2}+\frac{\alpha}{4}\right)(z k)^{4}+\ldots\right] L i_{\alpha-1}(\zeta) \\
& +\frac{1}{\Gamma(3-\alpha)}\left[-\frac{\alpha}{2}(z k)^{3}+\frac{\alpha}{4}(z k)^{4}+\ldots\right]
\end{aligned}
$$

where $L i_{\alpha-1}(\zeta)$ and $L i_{\alpha-2}(\zeta)$ with $0<\alpha<1$ denote the polylogarithm functions. By Lemma 2.7 with $p=\alpha-1, \alpha-2$, we have

$$
\begin{aligned}
\widetilde{w}(z)= & (z k)^{\alpha}+\frac{1}{\Gamma(3-\alpha)}\left(\frac{1}{2} \Gamma(3-\alpha)+\left(-1+\frac{\alpha}{2}\right) \Gamma(2-\alpha)\right)(z k)^{\alpha+1} \\
& +\frac{1}{\Gamma(3-\alpha)}\left(\frac{1}{4} \Gamma(3-\alpha)+\left(-\frac{1}{2}+\frac{\alpha}{4}\right) \Gamma(2-\alpha)\right)(z k)^{\alpha+2}+d_{2}(z k)^{3}+d_{3}(z k)^{3+\alpha}+\ldots \\
= & (z k)^{\alpha}+d_{2}(z k)^{3}+d_{3}(z k)^{3+\alpha}+\ldots,
\end{aligned}
$$

for some suitable constants $d_{2}, d_{3}, \ldots$

Together these estimates complete the proof of Lemma 2.5 .

Lemma 2.8. Let $\zeta=e^{-z k}$ and $z \in \Gamma_{k}$. Let $\mu(\zeta), z_{k}$ and $K(z)$ be defined as in (27), (25), (29), respectively. We have,

$$
\begin{aligned}
& \mu\left(e^{-z k}\right)-1=O\left((z k)^{3-\alpha}\right), \quad \text { as } z k \rightarrow 0 \\
& c|z| \leq\left|z_{k}\right| \leq C|z| \\
& \left\|K\left(z_{k}\right)-K(z)\right\| \leq C k^{3-\alpha}|z|^{2-\alpha} \\
& \left\|\mu(\zeta) K\left(z_{k}\right)-K(z)\right\| \leq C k^{3-\alpha}|z|^{2-\alpha}
\end{aligned}
$$


Proof: See the Appendix.

We now turn to the proof of Theorem 2.3. To prove Theorem 2.3, we need to show that $z_{k}^{\alpha} \in \Sigma_{\theta_{0}}$ for some $\theta_{0} \in(\pi / 2, \pi)$ where $z_{k}$ is defined in (25) below and $\theta_{0}$ is introduced in (2). We have the following lemma, [16, Lemma 3.7]

Lemma 2.9. Let $\theta>\pi / 2$ be close to $\pi / 2$. Let $z \in \Gamma_{k}$ with $\Gamma_{k}=\{z \in \Gamma:|\Im z| \leq \pi / k\}$ and $\Gamma=\{z$ : $|\arg z|=\theta\}$ (with $\Im z$ running from $-\infty$ to $\infty$ ). Let $z_{k}=\frac{\delta(\zeta)}{k}, \zeta=e^{-z k}$ be defined by (25). Then there exists $\theta_{0} \in(\pi / 2, \pi)$ such that

$$
z_{k}^{\alpha} \in \Sigma_{\theta_{0}}, \quad \text { for all } z \in \Sigma_{\theta}
$$

Remark 2.3. In Jin et al. [16, Lemma 3.7], the authors proved that for all $-\pi \leq \theta<\pi$, there exists $\theta_{0} \in(\pi / 2, \pi)$ such that $z_{k}^{\alpha} \in \Sigma_{\theta_{0}}$ for all $z \in \Sigma_{\theta}$. Actually in our analysis, we only need to show $z_{k}^{\alpha} \in \Sigma_{\theta_{0}}$ for all $z \in \Sigma_{\theta}$ for some $\theta>\pi / 2$ close to $\pi / 2$.

Proof of Theorem 2.3: $\quad$ Taking the Laplace transform in (4), we have,

$$
\hat{V}(z)=-z^{-1}\left(z^{\alpha}+A\right)^{-1} A u_{0},
$$

which implies that

$$
V(t)=-\frac{1}{2 \pi i} \int_{\Gamma} e^{z t} z^{-1}\left(z^{\alpha}+A\right)^{-1} A u_{0} d z,
$$

where $\Gamma=\Gamma_{\theta}=\{z:|\arg z|=\theta\}$, for some $\theta>\pi / 2$ and $\theta$ is close to $\pi / 2$.

Denote

$$
\sigma_{n}=\left\{\begin{array}{l}
1+a_{1}, \quad n=1 \\
1+a_{2}, \quad n=2 \\
1, \quad n \geq 3
\end{array}\right.
$$

The time discretization problem (11)-(14) can then be rewritten as

$$
k^{-\alpha} \sum_{j=1}^{n} w_{n-j} V^{j}+A V^{n}=\left(-A u_{0}\right) \sigma_{n} .
$$

Taking the summation with $n=1,2, \ldots$, we have

$$
\sum_{n=1}^{\infty}\left(k^{-\alpha} \sum_{j=1}^{n} w_{n-j} V^{j}\right) \zeta^{n}+\sum_{n=1}^{\infty}\left(A V^{n}\right) \zeta^{n}=\left(-A u_{0}\right)\left(\sum_{n=1}^{\infty} \sigma_{n} \zeta^{n}\right) .
$$

Using the following equality

$$
\sum_{n=1}^{\infty}\left(\sum_{j=1}^{n} w_{n-j} V^{j}\right) \zeta^{n}=\left(\sum_{j=0}^{\infty} w_{j} \zeta^{j}\right)\left(V^{1} \zeta^{1}+V^{2} \zeta^{2}+\ldots\right) .
$$

we have, with $\tilde{V}(\zeta)=\sum_{j=0}^{\infty} V^{j} \zeta^{j}$,

$$
k^{-\alpha} \widetilde{w}(\zeta) \widetilde{V}(\zeta)+A \widetilde{V}(\zeta)=\left(-A u_{0}\right)\left(\frac{\zeta}{1-\zeta}+a_{1} \zeta+a_{2} \zeta^{2}\right)
$$

which implies that

$$
\widetilde{V}(\zeta)+k^{\alpha} \widetilde{w}(\zeta)^{-1} A \widetilde{V}(\zeta)=k^{\alpha} \widetilde{w}(\zeta)^{-1}\left(-A u_{0}\right)\left(\frac{\zeta}{1-\zeta}+a_{1} \zeta+a_{2} \zeta^{2}\right)
$$


Denote $\delta(\zeta)^{\alpha}=\tilde{w}(\zeta)$, we have

$$
\widetilde{V}(\zeta)+\left(\frac{\delta(\zeta)}{k}\right)^{-\alpha} A \widetilde{V}(\zeta)=-\left(\frac{\delta(\zeta)}{k}\right)^{-\alpha}\left(\frac{\zeta}{1-\zeta}+a_{1} \zeta+a_{2} \zeta^{2}\right) A u_{0}
$$

With

$$
z_{k}=\frac{\delta(\zeta)}{k}, \quad \delta(\zeta)=\tilde{w}(\zeta)^{\frac{1}{\alpha}}
$$

we obtain

$$
\widetilde{V}(\zeta)=-\left(z_{k}^{\alpha}+A\right)^{-1}\left(\frac{\zeta}{1-\zeta}+a_{1} \zeta+a_{2} \zeta^{2}\right) A u_{0},
$$

By Taylor series expansion, we have, [23],

$$
\begin{aligned}
V^{n} & =-\frac{1}{2 \pi i} \int_{|\zeta|=\rho} \zeta^{-n-1}\left(\frac{\zeta}{1-\zeta}+a_{1} \zeta+a_{2} \zeta^{2}\right)\left(z_{k}^{\alpha}+A\right)^{-1} A u_{0} d \zeta \\
& =-\frac{1}{2 \pi i} \int_{|\zeta|=\rho} \zeta^{-n-1}\left(\frac{\zeta}{1-\zeta}+a_{1} \zeta+a_{2} \zeta^{2}\right) z_{k} z_{k}^{-1}\left(z_{k}^{\alpha}+A\right)^{-1} A u_{0} d \zeta
\end{aligned}
$$

Let $\zeta=e^{-z k}, z=\frac{1}{k} \ln \frac{1}{\rho}+i\left(-\frac{\theta}{k}\right),|\theta| \leq \pi$, we have

$$
V^{n}=\frac{1}{2 \pi i} \int_{\Gamma_{k}} e^{t_{n} z}\left(\frac{\zeta}{1-\zeta}+a_{1} \zeta+a_{2} \zeta^{2}\right) \delta(\zeta) z_{k}^{-1}\left(z_{k}^{\alpha}+A\right)^{-1} A u_{0} d z,
$$

where $\Gamma_{k}=\{z \in \Gamma:|\Im z| \leq \pi / k\}$. For the details of the notation $\Gamma_{k}$, see the proof of Lemma 3.2 in [16]. Denote

$$
\mu(\zeta)=\left(\frac{\zeta}{1-\zeta}+a_{1} \zeta+a_{2} \zeta^{2}\right) \delta(\zeta)
$$

we get

$$
V^{n}=\frac{1}{2 \pi i} \int_{\Gamma_{k}} e^{t_{n} z} \mu(\zeta) z_{k}^{-1}\left(z_{k}^{\alpha}+A\right)^{-1} A u_{0} d z .
$$

Subtracting (23) from (28), we have

$$
\begin{aligned}
V\left(t_{n}\right)-V^{n}= & \frac{1}{2 \pi i} \int_{\Gamma_{k}} e^{t_{n} z}\left(\mu(\zeta) z_{k}^{-1}\left(z_{k}^{\alpha}+A\right)^{-1}-z^{-1}\left(z^{\alpha}+A\right)^{-1}\right) A u_{0} d z \\
& +\frac{1}{2 \pi i} \int_{\Gamma \backslash \Gamma_{k}} e^{t_{n} z} z^{-1}\left(z^{\alpha}+A\right)^{-1} A u_{0} d z \\
= & I+I I .
\end{aligned}
$$

Denote

$$
K(z)=z^{-1}\left(z^{\alpha}+A\right)^{-1} A .
$$

For $I$, we have, by (21),

$$
\begin{aligned}
\|I\| & \leq \frac{1}{2 \pi} \int_{\Gamma_{k}}\left|e^{t_{n} z}\right|\left\|\mu(\zeta) K\left(z_{k}\right)-K(z)\left|\left\|u_{0}\right\|\right| d z\left|\leq \frac{1}{2 \pi} \int_{\Gamma_{k}}\right| e^{t_{n} z}\right\| C\left(k^{3-\alpha}|z|^{2-\alpha}\right)\left\|u_{0}\right\||d z| \\
& \leq C k^{3-\alpha} \int_{0}^{\infty} e^{-c t_{n} r}\left(r t_{n}\right)^{2-\alpha} t_{n}^{\alpha-2} d\left(r t_{n}\right) t_{n}^{-1}\left\|u_{0}\right\| \leq C k^{3-\alpha} t_{n}^{\alpha-3}\left\|u_{0}\right\| .
\end{aligned}
$$

For $I I$, we have, by (3) and noting that $\left(z^{\alpha}+A\right)^{-1} A=I-z^{\alpha}\left(z^{\alpha}+A\right)^{-1}$,

$$
\left\|\left(z^{\alpha}+A\right)^{-1} A\right\|=\left\|I-z^{\alpha}\left(z^{\alpha}+A\right)^{-1}\right\| \leq\|I\|+\left\|z^{\alpha}\left(z^{\alpha}+A\right)^{-1}\right\| \leq 1+M \leq C .
$$


Thus, with some constant $c>0$,

$$
\begin{aligned}
\|I I\| & \leq \frac{1}{2 \pi} \int_{\Gamma \backslash \Gamma_{k}}\left|e^{t_{n} z}\right|\left\|z^{-1}\left(z^{\alpha}+A\right)^{-1} A\right\|\left\|u_{0}\right\||d z| \leq C \int_{\frac{1}{k}}^{\infty} e^{-c t_{n}|z|}|z|^{-1}|d z|\left\|u_{0}\right\| \\
& \leq C \int_{\frac{1}{k}}^{\infty} e^{-c t_{n}|z|}|z|^{-(3-\alpha)}|z|^{-\alpha+2}|d z|\left\|u_{0}\right\| \leq C k^{3-\alpha} \int_{\frac{1}{k}}^{\infty} e^{-c t_{n}|z|}|z|^{-\alpha+2}|d z|\left\|u_{0}\right\| \\
& \leq C k^{3-\alpha} \int_{\frac{1}{k}}^{\infty} e^{-c t_{n}|z|}\left(t_{n} r\right)^{-\alpha+2} d\left(r t_{n}\right)\left(t_{n}\right)^{\alpha-2}\left(t_{n}\right)^{-1}\left\|u_{0}\right\||d z| \\
& \leq C k^{3-\alpha} t_{n}^{\alpha-3} \int_{0}^{\infty} e^{-c t_{n} r}\left(t_{n} r\right)^{-\alpha+2} d\left(r t_{n}\right)\left\|u_{0}\right\||d z| \leq C k^{3-\alpha} t_{n}^{\alpha-3}\left\|u_{0}\right\| .
\end{aligned}
$$

The proof of Theorem 2.3 is now complete.

Remark 2.4. We remark that assuming $u_{0} \in \mathcal{D}(A)$ rather than $u_{0} \in L_{2}(\Omega)$ reduces the singular behavior of the error bound at $t=0$. We can prove the convergence order $O\left(k^{3-\alpha}\right), 0<\alpha<1$ similarly, see Lubich et al. [23, p.16]

\section{The inhomogeneous problem}

In this section we will consider the time stepping method for solving the inhomogeneous problem (1) based on the time stepping method introduced in Section 2 for the homogeneous problem.

Let $u(t)-u_{0}=V(t)$. Then (1) is equivalent to

$$
{ }_{0}^{C} D_{t}^{\alpha} V(t)+A V(t)=-A u_{0}+f(t), 0<t \leq T, \quad \text { with } V(0)=0 .
$$

With $V^{n} \approx V\left(t_{n}\right), n=0,1,2, \ldots, N$ as in Section 2, we define the following time discretization scheme for solving (30), with $V^{0}=0$,

$$
\begin{aligned}
& k^{-\alpha} \sum_{j=1}^{n} w_{n-j} V^{j}+A V^{n}=-A u_{0}+f\left(t_{n}\right)+a_{1}\left(-A u_{0}+f(0)\right)+b_{1} k f^{\prime}(0), n=1, \\
& k^{-\alpha} \sum_{j=1}^{n} w_{n-j} V^{j}+A V^{n}=-A u_{0}+f\left(t_{n}\right)+a_{2}\left(-A u_{0}+f(0)\right)+b_{2} k f^{\prime}(0), n=1, \\
& k^{-\alpha} \sum_{j=1}^{n} w_{n-j} V^{j}+A V^{j}=-A u_{0}+f\left(t_{n}\right), n=2,3, \ldots, N,
\end{aligned}
$$

where $w_{j}, j=0,1,2, \ldots$ are defined by (16). Here the coefficients $a_{1}, a_{2}, b_{1}, b_{2}$ are defined by

$$
a_{1}=\frac{11}{12}, a_{2}=-\frac{5}{12}, b_{1}=\frac{1}{12}, b_{2}=0
$$

which are determined by Theorem 2.3 and Lemma 3.2 below.

Theorem 3.1. Let $V\left(t_{n}\right)$ and $V^{n}$ be the solutions of (30) and (31)-(33), respectively. Let $u_{0} \in H=L_{2}(\Omega)$. Then we have, with $0<\alpha<1$,

$$
\left\|V\left(t_{n}\right)-V^{n}\right\| \leq C k^{3-\alpha}\left(t_{n}^{\alpha-3}\left\|u_{0}\right\|+t_{n}^{2 \alpha-3}\|f(0)\|+t_{n}^{2 \alpha-2}\left\|f^{\prime}(0)\right\|+t_{n}^{2 \alpha-1}\left\|f^{\prime \prime}(0)\right\|+\int_{0}^{t_{n}}\left(t_{n}-s\right)^{2 \alpha-1}\left\|f^{\prime \prime \prime}(s)\right\| d s\right) .
$$


To prove Theorem 3.1, we need the following lemmas.

Lemma 3.2. Let $z_{k}$ be defined as in (25). Let $b_{1}=\frac{1}{12}, b_{2}=0$. Then we have

$$
\left\|\left(z^{\alpha}+A\right)^{-1} z^{-2}-\left(z_{k}^{\alpha}+A\right)^{-1}\left(\sum_{n=1}^{\infty} n \zeta^{n}+b_{1} \zeta+b_{2} \zeta^{2}\right) k^{2}\right\| \leq C k^{3-\alpha}|z|^{1-2 \alpha} .
$$

Proof: The proof is similar as the proof of Jin et al. [18, Lemma C.1.]. We omit the proof here.

Lemma 3.3. Let $z_{k}$ be defined as in (25), We have

$$
\left\|\left(z^{\alpha}+A\right)^{-1} z^{-3}-\left(z_{k}^{\alpha}+A\right)^{-1}\left(k \sum_{n=1}^{\infty} \frac{t_{n}^{2}}{2 !} \zeta^{n}\right)\right\| \leq C k^{3-\alpha}|z|^{-2 \alpha} .
$$

Proof: We have

$$
\begin{aligned}
& \left\|\left(z^{\alpha}+A\right)^{-1} z^{-3}-\left(z_{k}^{\alpha}+A\right)^{-1}\left(k \sum_{n=1}^{\infty} t_{n} \zeta^{n}\right)\right\| \\
& \leq\left\|\left(z^{\alpha}+A\right)^{-1} z^{-3}-\left(z_{k}^{\alpha}+A\right)^{-1} z_{k}^{-3}\right\|+\left\|\left(z_{k}^{\alpha}+A\right)^{-1} z_{k}^{-3}\left(1-z_{k}^{3} k \sum_{n=1}^{\infty} \frac{t_{n}^{2}}{2 !} \zeta^{n}\right)\right\| .
\end{aligned}
$$

It is easy to show that

$$
\left\|1-z_{k}^{3} k \sum_{n=1}^{\infty} \frac{t_{n}^{2}}{2 !} \zeta^{n}\right\| \leq C|z k|^{3-\alpha} .
$$

The rest of the proof of Lemma 3.3 follows from the arguments in the proof of (20)-(21).

Proof of Theorem 3.1: $\quad$ The proof is similar to the arguments developed in Jin et al. [17], [18] for considering the time stepping methods for solving (1) with $f \neq 0$.

Denote

$$
f(t)=f(0)+f^{\prime}(0) t+R(t), \quad R(t)=\frac{t^{2}}{2 !} f^{\prime \prime}(0)+\left(\frac{t^{2}}{2 !} * f^{\prime \prime \prime}\right)(t) .
$$

Here $f * g$ denotes the convolution of $f$ and $g$.

Taking the Laplace transform in (30), we have

$$
z^{\alpha} \hat{V}(z)+A \hat{V}(z)=\left(-A u_{0}+f(0)\right) z^{-1}+f^{\prime}(0) z^{-2}+\hat{R}(z)
$$

which implies that

$$
V(t)=\frac{1}{2 \pi i} \int_{\Gamma} e^{z t}\left(\left(z^{\alpha}+A\right)^{-1} z^{-1}\left(-A u_{0}+f(0)\right)+\left(z^{\alpha}+A\right)^{-1} z^{-2} f^{\prime}(0)+\left(z^{\alpha}+A\right)^{-1} \hat{R}(z)\right) d z
$$

Taking the discrete Laplace transform in (31)-(33), we have

$$
\begin{aligned}
\sum_{n=1}^{\infty}\left(k^{-\alpha} \sum_{j=1}^{n} w_{n-j} V^{j}\right) \zeta^{n} & +\sum_{n=1}^{\infty}\left(A V^{n}\right) \zeta^{n}=\left(-A u_{0}+f(0)\right)\left(a_{1} \zeta+a_{2} \zeta^{2}+\frac{\zeta}{1-\zeta}\right) \\
& +\sum_{n=1}^{\infty}\left(t_{n} f^{\prime}(0)\right) \zeta^{n}+\sum_{n=1}^{\infty} R\left(t_{n}\right) \zeta^{n}+b_{1} k f^{\prime}(0) \zeta+b_{2} k f^{\prime}(0) \zeta^{2}
\end{aligned}
$$


which implies that

$$
\begin{aligned}
V^{n}= & -\frac{1}{2 \pi i} \int_{\Gamma_{k}} e^{z t_{n}}\left(\left(z_{k}^{\alpha}+A\right)^{-1} z_{k}^{-1}\left(A u_{0}-f(0)\right)\right)\left(z_{k} k\left(a_{1} \zeta+a_{2} \zeta^{2}+\frac{\zeta}{1-\zeta}\right)\right) d z \\
& -\frac{1}{2 \pi i} \int_{\Gamma_{k}} e^{z t_{n}}\left(\left(z_{k}^{\alpha}+A\right)^{-1} z_{k}^{-1} f^{\prime}(0)\right)\left(-z_{k} k^{2}\left(\sum_{n=1}^{\infty} n \zeta^{n}+b_{1} \zeta+b_{2} \zeta^{2}\right)\right) d z \\
& -\frac{1}{2 \pi i} \int_{\Gamma_{k}} e^{z t_{n}}\left(\left(z_{k}^{\alpha}+A\right)^{-1} z_{k}^{-1}\right)\left(-z_{k} k\left(\sum_{n=1}^{\infty} R\left(t_{n}\right) \zeta^{n}\right)\right) d z,
\end{aligned}
$$

where $\mu(\zeta)$ and $z_{k}$ are defined by (27) and (25), respectively. Thus we have

$$
V\left(t_{n}\right)-V^{n}=I_{1}+I_{2}+I_{3},
$$

where

$$
\begin{aligned}
I_{1}= & \frac{1}{2 \pi i} \int_{\Gamma / \Gamma_{k}} e^{z t_{n}}\left(z^{\alpha}+A\right)^{-1} z^{-1}\left(-A u_{0}+f(0)\right) d z \\
& +\frac{1}{2 \pi i} \int_{\Gamma_{k}} e^{z t_{n}}\left(\left(z^{\alpha}+A\right)^{-1} z^{-1}-\left(z_{k}^{\alpha}+A\right)^{-1} z_{k}^{-1} \mu\left(e^{-z k}\right)\right)\left(-A u_{0}+f(0)\right) d z \\
I_{2}= & \frac{1}{2 \pi i} \int_{\Gamma} e^{z t_{n}}\left(\left(z^{\alpha}+A\right)^{-1} z^{-2}\right) f^{\prime}(0) d z \\
& -\frac{1}{2 \pi i} \int_{\Gamma_{k}} e^{z t_{n}}\left(\left(z_{k}^{\alpha}+A\right)^{-1}\left(\sum_{n=1}^{\infty} n \zeta^{n}+b_{1} \zeta+b_{2} \zeta^{2}\right) k^{2}\right) f^{\prime}(0) d z \\
I_{3}= & \frac{1}{2 \pi i} \int_{\Gamma} e^{z t_{n}}\left(\left(z^{\alpha}+A\right)^{-1} z^{-1}\right)(z \hat{R}(z)) d z \\
& -\frac{1}{2 \pi i} \int_{\Gamma_{k}} e^{z t_{n}}\left(\left(z_{k}^{\alpha}+A\right)^{-1} z_{k}^{-1}\left(z_{k} k \sum_{n=1}^{\infty} R\left(t_{n}\right) \zeta^{n}\right)\right) d z .
\end{aligned}
$$

For $I_{1}$, we have, following the same argument as in the proof of Theorem 2.3,

$$
\left\|I_{1}\right\| \leq C k^{2-\alpha} t_{n}^{\alpha-2}\left\|u_{0}\right\|+C k^{2-\alpha} t_{n}^{2 \alpha-2}\|f(0)\| .
$$

For $I_{2}$, we have

$$
\begin{aligned}
I_{2}= & \frac{1}{2 \pi i} \int_{\Gamma / \Gamma_{k}} e^{z t_{n}}\left(\left(z^{\alpha}+A\right)^{-1} z^{-2}\right) f^{\prime}(0) d z \\
& +\frac{1}{2 \pi i} \int_{\Gamma_{k}} e^{z t_{n}}\left(\left(z^{\alpha}+A\right)^{-1} z^{-2}-\left(z_{k}^{\alpha}+A\right)^{-1}\left(\sum_{n=1}^{\infty} n \zeta^{n}+b_{1} \zeta+b_{2} \zeta^{2}\right) k^{2}\right) f^{\prime}(0) d z \\
= & I_{21}+I_{22} .
\end{aligned}
$$

For $I_{21}$, we have, following the arguments as in the proof of Theorem 2.3,

$$
\left\|I_{21}\right\| \leq C k^{3-\alpha} t_{n}^{2 \alpha-2}\left\|f^{\prime}(0)\right\| .
$$

For $I_{22}$, we have

$$
\left\|I_{22}\right\| \leq C\left\|\int_{\Gamma_{k}} e^{z t_{n}}\left(\left(z^{\alpha}+A\right)^{-1} z^{-2}-\left(z_{k}^{\alpha}+A\right)^{-1}\left(\sum_{n=1}^{\infty} n \zeta^{n}+b_{1} \zeta+b_{2} \zeta^{2}\right) k^{2}\right) d z\right\|\left\|f^{\prime}(0)\right\| .
$$

By Lemma 3.2, we have

$$
\left\|I_{22}\right\| \leq C \int_{\Gamma_{k}} e^{-c t_{n} r} k^{3-\alpha} r^{1-2 \alpha} d r\left\|f^{\prime}(0)\right\| \leq C k^{3-\alpha} t_{n}^{2 \alpha-2}\left\|f^{\prime}(0)\right\| .
$$


Thus we get

$$
\left\|I_{2}\right\| \leq C k^{3-\alpha} t_{n}^{2 \alpha-2}\left\|f^{\prime}(0)\right\| .
$$

For $I_{3}$, we may write $I_{3}$ as

$$
I_{3}=I_{3}^{1}+I_{3}^{2}
$$

Here

$$
\begin{aligned}
I_{3}^{1}= & \frac{1}{2 \pi i} \int_{\Gamma} e^{z t_{n}}\left(\left(z^{\alpha}+A\right)^{-1} z^{-1}\right)\left(z \hat{R}^{1}(z)\right) d z \\
& -\frac{1}{2 \pi i} \int_{\Gamma_{k}} e^{z t_{n}}\left(\left(z_{k}^{\alpha}+A\right)^{-1} z_{k}^{-1}\left(z_{k} k \sum_{n=1}^{\infty} R^{1}\left(t_{n}\right) \zeta^{n}\right)\right) d z \\
I_{3}^{2}= & \frac{1}{2 \pi i} \int_{\Gamma} e^{z t_{n}}\left(\left(z^{\alpha}+A\right)^{-1} z^{-1}\right)\left(z \hat{R}^{2}(z)\right) d z \\
& -\frac{1}{2 \pi i} \int_{\Gamma_{k}} e^{z t_{n}}\left(\left(z_{k}^{\alpha}+A\right)^{-1} z_{k}^{-1}\left(z_{k} k \sum_{n=1}^{\infty} R^{2}\left(t_{n}\right) \zeta^{n}\right)\right) d z,
\end{aligned}
$$

where

$$
R(t)=\frac{t^{2}}{2 !} f^{\prime \prime}(0)+\left(\frac{t^{2}}{2 !} * f^{\prime \prime \prime}\right)(t)=: R^{1}(t)+R^{2}(t) .
$$

For $I_{3}^{1}$, we have

$$
\begin{aligned}
\left\|I_{3}^{1}\right\|= & \| \frac{1}{2 \pi i} \int_{\Gamma} e^{z t_{n}}\left(\left(z^{\alpha}+A\right)^{-1} z^{-3}\right) d z f^{\prime \prime}(0) \\
& -\frac{1}{2 \pi i} \int_{\Gamma_{k}} e^{z t_{n}}\left(\left(z_{k}^{\alpha}+A\right)^{-1}\left(k \sum_{n=1}^{\infty} R^{1}\left(t_{n}\right) \zeta^{n}\right)\right) d z f^{\prime \prime}(0) \| \\
= & \| \frac{1}{2 \pi i} \int_{\Gamma / \Gamma_{k}} e^{z t_{n}}\left(\left(z^{\alpha}+A\right)^{-1} z^{-3}\right) d z f^{\prime \prime}(0) \\
& -\frac{1}{2 \pi i} \int_{\Gamma_{k}} e^{z t_{n}}\left(\left(z^{\alpha}+A\right)^{-1} z^{-3}-\left(z_{k}^{\alpha}+A\right)^{-1}\left(k \sum_{n=1}^{\infty} R^{1}\left(t_{n}\right) \zeta^{n}\right)\right) d z f^{\prime \prime}(0) \| .
\end{aligned}
$$

By Lemma 3.3, we have

$$
\left\|I_{3}^{1}\right\| \leq C k^{3-\alpha} t_{n}^{2 \alpha-1}\left\|f^{\prime \prime}(0)\right\| .
$$

For $I_{3}^{2}$, we have, following the arguments as in Jin et al. [17], [18],

$$
\left\|I_{3}^{2}\right\| \leq C k^{3-\alpha} \int_{0}^{t_{n}}\left(t_{n}-s\right)^{2 \alpha-1}\left\|f^{\prime \prime \prime}(s)\right\| d s .
$$

Together these estimates complete the proof of Theorem 3.1 .

\section{Numerical example}

In this section, we will consider the experimentally determined convergence rates of the numerical method (11)-(14) for smooth and nonsmooth initial data. We only show the numerical results for the homogeneous problem here. Similarly we may consider the numerical results for the inhomogeneous problem with $f \neq 0$ as discussed in Jin et al. [17], [18]. 
Example 4.1. Consider, with $0<\alpha<1$,

$$
\begin{aligned}
& { }_{0}^{C} D_{t}^{\alpha} u(x, t)-\frac{\partial^{2} u(x, t)}{\partial x^{2}}=0, \quad 0<x<1, \quad 0<t \leq T, \\
& u(0, t)=u(1, t)=0, \\
& u(x, 0)=u_{0}(x),
\end{aligned}
$$

where $(a) u_{0}(x)=\sin (2 \pi x)$ (smooth data) and (b) $u_{0}(x)=\chi_{[0,1 / 2]}$ (nonsmooth data).

Let $0<t_{0}<t_{1}<\cdots<t_{N}=T$ be the time partition and $k$ the time step size. Let $N_{h}$ be a positive integer. Let $0=x_{0}<x_{1}<x_{2}<\cdots<x_{N_{h}}=1$ be the space partition and $h$ the space step size. The space is discretized by using the standard linear finite element method.

We first consider the L1 scheme (7) and the convergence rate was proved to be $O(k)$ for both smooth and nonsmooth data in [16]. To observe this convergence rate, we first calculate the reference solution $u_{\text {ref }}(t)$ at $T=1$ with $h_{r e f}=2^{-6}$ and $k_{r e f}=2^{-10}$. We then use $h=2^{-6}$ and $k=\kappa * k_{\text {ref }}$ with $\kappa=\left[2^{2}, 2^{3}, 2^{4}, 2^{5}, 2^{6}\right]$ to obtain the approximate solution at $u(T)$. We obtain the following results which are consistent with the Table 1 in [16]. The convergence rate indeed is almost $O(k)$ for the different $\alpha \in(0,1)$ in both smooth and nonsmooth data cases.

\begin{tabular}{cccccccc}
$\alpha$ & & $k=2^{-8}$ & $k=2^{-7}$ & $k=2^{-6}$ & $k=2^{-5}$ & $k=2^{-4}$ & Rate \\
\hline 0.1 & $(a)$ & $2.12 \mathrm{e}-6$ & $4.96 \mathrm{e}-6$ & $1.06 \mathrm{e}-5$ & $2.22 \mathrm{e}-5$ & $4.56 \mathrm{e}-5$ & 1.1063 \\
& $(b)$ & $5.51 \mathrm{e}-6$ & $1.27 \mathrm{e}-5$ & $2.74 \mathrm{e}-5$ & $5.70 \mathrm{e}-5$ & $1.17 \mathrm{e}-4$ & 1.1063 \\
0.3 & $(a)$ & $5.61 \mathrm{e}-6$ & $1.30 \mathrm{e}-5$ & $2.80 \mathrm{e}-5$ & $5.85 \mathrm{e}-5$ & $1.21 \mathrm{e}-4$ & 1.1100 \\
& $(b)$ & $1.43 \mathrm{e}-5$ & $3.33 \mathrm{e}-5$ & $7.18 \mathrm{e}-5$ & $1.48 \mathrm{e}-4$ & $3.09 \mathrm{e}-4$ & 1.1099 \\
0.8 & $(a)$ & $7.81 \mathrm{e}-6$ & $1.85 \mathrm{e}-5$ & $4.03 \mathrm{e}-5$ & $8.57 \mathrm{e}-5$ & $1.82 \mathrm{e}-4$ & 1.1359 \\
& $(b)$ & $1.98 \mathrm{e}-5$ & $4.66 \mathrm{e}-5$ & $1.02 \mathrm{e}-4$ & $2.16 \mathrm{e}-4$ & $4.59 \mathrm{e}-4$ & 1.1350 \\
0.9 & $(a)$ & $5.41 \mathrm{e}-6$ & $1.28 \mathrm{e}-5$ & $2.84 \mathrm{e}-5$ & $6.21 \mathrm{e}-5$ & $1.40 \mathrm{e}-5$ & 1.1766 \\
& $(b)$ & $1.34 \mathrm{e}-5$ & $3.20 \mathrm{e}-5$ & $7.08 \mathrm{e}-5$ & $1.55 \mathrm{e}-5$ & $3.49 \mathrm{e}-5$ & 1.1757 \\
\hline
\end{tabular}

Table 1: Time convergence rates with the different $\alpha \in(0,1)$ for the L1 scheme (7) in Example 4.1

We next consider the modified L1 scheme (8)-(10) which has the convergence rate $O\left(k^{2-\alpha}\right)$ for both smooth and nonsmooth data. Using the same notations and the same initial data as in Table 1, we found, in Table 2, that the modified L1 scheme has the better accuracy than the L1 scheme. When $\alpha<1 / 2$, the convergence rates are almost 2 which is better than the theoretical results $2-\alpha$. However when $\alpha>1 / 2$, the convergence rates are almost $2-\alpha$ as we expected.

Finally we consider the higher order numerical method (11)-(14) which has the convergence rate $O\left(k^{3-\alpha}\right)$ for both smooth and nonsmooth data. Using the same notations and the same initial data as in Tables 1 and 2, we found, in Table 3, that the higher order numerical method (11)-(14) has the better accuracy than the L1 scheme and the modified L1 scheme as we expected. We observe that when $\alpha<1 / 2$, the convergence rates are almost 3 which is better than the theoretical results $3-\alpha$. However when $\alpha>1 / 2$, the convergence rates are almost $3-\alpha$ as we expected.

Example 4.2. In this example, we will consider, with $0<\alpha<1$,

$$
\begin{aligned}
& { }_{0}^{C} D_{t}^{\alpha} u(x, t)-\frac{1}{4 \pi^{2}} \frac{\partial^{2} u(x, t)}{\partial x^{2}}=0, \quad 0<x<1, \quad 0<t \leq T, \\
& u(0, t)=u(1, t)=0, \\
& u(x, 0)=u_{0}(x),
\end{aligned}
$$




\begin{tabular}{cccccccc}
$\alpha$ & & $k=2^{-8}$ & $k=2^{-7}$ & $k=2^{-6}$ & $k=2^{-5}$ & $k=2^{-4}$ & Rate \\
\hline 0.1 & $(a)$ & $7.12 \mathrm{e}-9$ & $3.01 \mathrm{e}-8$ & $1.25 \mathrm{e}-7$ & $5.17 \mathrm{e}-7$ & $2.19 \mathrm{e}-6$ & 2.0674 \\
& $(b)$ & $1.82 \mathrm{e}-8$ & $7.81 \mathrm{e}-8$ & $3.22 \mathrm{e}-7$ & $1.33 \mathrm{e}-6$ & $5.65 \mathrm{e}-6$ & 2.0668 \\
0.3 & $(a)$ & $1.31 \mathrm{e}-8$ & $6.42 \mathrm{e}-8$ & $2.91 \mathrm{e}-7$ & $1.30 \mathrm{e}-6$ & $5.89 \mathrm{e}-6$ & 2.1914 \\
& $(b)$ & $4.23 \mathrm{e}-8$ & $1.71 \mathrm{e}-7$ & $7.61 \mathrm{e}-7$ & $3.39 \mathrm{e}-6$ & $1.53 \mathrm{e}-5$ & 2.1839 \\
0.8 & $(a)$ & $7.91 \mathrm{e}-7$ & $2.01 \mathrm{e}-6$ & $4.62 \mathrm{e}-6$ & $9.81 \mathrm{e}-6$ & $1.78 \mathrm{e}-5$ & 1.1223 \\
& $(b)$ & $1.96 \mathrm{e}-6$ & $4.96 \mathrm{e}-6$ & $1.14 \mathrm{e}-5$ & $2.42 \mathrm{e}-5$ & $4.41 \mathrm{e}-5$ & 1.1230 \\
0.9 & $(a)$ & $1.41 \mathrm{e}-6$ & $3.45 \mathrm{e}-6$ & $7.78 \mathrm{e}-6$ & $1.68 \mathrm{e}-5$ & $3.48 \mathrm{e}-5$ & 1.1573 \\
& $(b)$ & $3.47 \mathrm{e}-6$ & $8.51 \mathrm{e}-6$ & $1.92 \mathrm{e}-5$ & $4.16 \mathrm{e}-5$ & $8.59 \mathrm{e}-5$ & 1.1572 \\
\hline
\end{tabular}

Table 2: Time convergence rates with the different $\alpha \in(0,1)$ for the modified L1 scheme (8)-(10) in Example 4.1

\begin{tabular}{cccccccc}
$\alpha$ & & $k=2^{-8}$ & $k=2^{-7}$ & $k=2^{-6}$ & $k=2^{-5}$ & $k=2^{-4}$ & Rate \\
\hline 0.1 & $(a)$ & $8.45 \mathrm{e}-11$ & $7.38 \mathrm{e}-10$ & $6.09 \mathrm{e}-9$ & $5.11 \mathrm{e}-8$ & $4.52 \mathrm{e}-7$ & 3.0962 \\
& $(b)$ & $1.92 \mathrm{e}-10$ & $1.68 \mathrm{e}-9$ & $1.39 \mathrm{e}-8$ & $1.16 \mathrm{e}-7$ & $1.03 \mathrm{e}-6$ & 3.0974 \\
0.3 & $(a)$ & $2.78 \mathrm{e}-10$ & $2.36 \mathrm{e}-9$ & $1.95 \mathrm{e}-8$ & $1.64 \mathrm{e}-7$ & $1.46 \mathrm{e}-6$ & 3.0909 \\
& $(b)$ & $6.19 \mathrm{e}-10$ & $5.26 \mathrm{e}-9$ & $4.36 \mathrm{e}-8$ & $3.69 \mathrm{e}-7$ & $3.30 \mathrm{e}-6$ & 3.0946 \\
0.8 & $(a)$ & $6.34 \mathrm{e}-10$ & $1.38 \mathrm{e}-9$ & $6.28 \mathrm{e}-9$ & $1.39 \mathrm{e}-7$ & $1.71 \mathrm{e}-6$ & 2.6496 \\
& $(b)$ & $1.64 \mathrm{e}-8$ & $7.33 \mathrm{e}-8$ & $3.01 \mathrm{e}-7$ & $1.06 \mathrm{e}-6$ & $2.06 \mathrm{e}-6$ & 2.1436 \\
0.9 & $(a)$ & $1.15 \mathrm{e}-9$ & $4.16 \mathrm{e}-9$ & $9.81 \mathrm{e}-9$ & $3.03 \mathrm{e}-8$ & $8.32 \mathrm{e}-7$ & 2.3756 \\
& $(b)$ & $4.11 \mathrm{e}-8$ & $1.82 \mathrm{e}-7$ & $7.65 \mathrm{e}-7$ & $3.09 \mathrm{e}-6$ & $1.11 \mathrm{e}-5$ & 2.0202 \\
\hline
\end{tabular}

Table 3: Time convergence rates with the different $\alpha \in(0,1)$ for $(11)$-(14) in Example 4.1

where $u_{0}(x)=\sin (2 \pi x)$ and the exact solution has the form

$$
u(x, t)=E_{\alpha, 1}\left(-t^{\alpha}\right) \sin (2 \pi x) .
$$

Here $E_{\alpha, 1}(z)$ denotes the Mittag-Leffler function defined by [30]

$$
E_{\alpha, 1}(z)=\sum_{k=0}^{\infty} \frac{z^{k}}{\Gamma(\alpha k+1)}, \quad \alpha>0
$$

We use the same notations as in Example 4.1 and the convergence rates are estimated by using the reference solution. In Table 4, we observe that the convergence rate for the L1 scheme (7) indeed is almost $O(k)$ for the different $\alpha \in(0,1)$.

We next consider the higher order numerical method (11)-(14) which has the convergence rate $O\left(k^{3-\alpha}\right)$ for both smooth and nonsmooth data. Using the same notations as in Table 4, we indeed observe the convergence rates are almost $O\left(k^{3-\alpha}\right)$ in Table 5 for the scheme (11)-(14).

Finally we consider the importance of the parameters $a_{1}, a_{2}$ in the scheme (11)-(14). To see this, we calculate the approximate solutions $V^{n}, n=1,2$ in the first two time levels $t_{1}$ and $t_{2}$ by using the exact solution (36) which can be evaluated by using the Mittag-Leffler MATLAB function "mlf.m" and then we obtain the approximate solutions $V^{n}, n=3,4, \ldots, N$ by using the scheme (13). In Table 6 , we obtain the convergence rates by using the reference solution as above and we see that the convergence rates are only $O(k)$. In other words, the parameters $a_{1}, a_{2}$ in the scheme (11)-(14) are very important for improving the convergence rates of the scheme. Even if we use the exact solutions in the first two time levels, the 


\begin{tabular}{ccccccc}
$\alpha$ & $k=2^{-8}$ & $k=2^{-7}$ & $k=2^{-6}$ & $k=2^{-5}$ & $k=2^{-4}$ & Rate \\
\hline 0.1 & $2.60 \mathrm{e}-5$ & $6.07 \mathrm{e}-5$ & $1.30 \mathrm{e}-4$ & $2.70 \mathrm{e}-4$ & $5.52 \mathrm{e}-4$ & 1.1017 \\
0.3 & $8.02 \mathrm{e}-5$ & $1.87 \mathrm{e}-4$ & $4.02 \mathrm{e}-4$ & $8.32 \mathrm{e}-4$ & $1.70 \mathrm{e}-3$ & 1.1012 \\
0.8 & $2.65 \mathrm{e}-4$ & $6.19 \mathrm{e}-4$ & $1.33 \mathrm{e}-3$ & $2.76 \mathrm{e}-3$ & $5.65 \mathrm{e}-3$ & 1.1032 \\
0.9 & $3.20 \mathrm{e}-4$ & $7.48 \mathrm{e}-4$ & $1.61 \mathrm{e}-3$ & $3.35 \mathrm{e}-3$ & $6.93 \mathrm{e}-3$ & 1.1091 \\
\hline
\end{tabular}

Table 4: Time convergence rates with the different $\alpha \in(0,1)$ for the L1 scheme $(7)$ by using the reference solution in Example 4.2

\begin{tabular}{ccccccc}
$\alpha$ & $k=2^{-8}$ & $k=2^{-7}$ & $k=2^{-6}$ & $k=2^{-5}$ & $k=2^{-4}$ & Rate \\
\hline 0.1 & $7.79 \mathrm{e}-10$ & $6.97 \mathrm{e}-9$ & $5.74 \mathrm{e}-8$ & $4.79 \mathrm{e}-7$ & $4.19 \mathrm{e}-6$ & 3.0987 \\
0.3 & $1.73 \mathrm{e}-9$ & $1.50 \mathrm{e}-8$ & $1.30 \mathrm{e}-7$ & $1.13 \mathrm{e}-6$ & $1.03 \mathrm{e}-5$ & 3.1373 \\
0.8 & $2.99 \mathrm{e}-8$ & $1.69 \mathrm{e}-7$ & $1.00 \mathrm{e}-6$ & $6.57 \mathrm{e}-6$ & $4.86 \mathrm{e}-5$ & 2.6671 \\
0.9 & $3.25 \mathrm{e}-7$ & $1.49 \mathrm{e}-6$ & $6.78 \mathrm{e}-6$ & $3.20 \mathrm{e}-5$ & $1.64 \mathrm{e}-4$ & 2.2445 \\
\hline
\end{tabular}

Table 5: Time convergence rates with the different $\alpha \in(0,1)$ for (11)-(14) by using the reference solution in Example 4.2

convergence rates can not be improved by using the scheme (13). However, if we use the scheme (11)-(14) to calculate all $V^{n}, n=1,2, \ldots, N$, then the convergence rates can be improved, i.e., Table 5 .

\begin{tabular}{ccccccc}
$\alpha$ & $k=2^{-8}$ & $k=2^{-7}$ & $k=2^{-6}$ & $k=2^{-5}$ & $k=2^{-4}$ & Rate \\
\hline 0.1 & $2.18 \mathrm{e}-5$ & $5.11 \mathrm{e}-5$ & $1.10 \mathrm{e}-4$ & $2.28 \mathrm{e}-4$ & $4.67 \mathrm{e}-4$ & 1.1047 \\
0.3 & $3.66 \mathrm{e}-5$ & $8.77 \mathrm{e}-5$ & $1.93 \mathrm{e}-4$ & $4.12 \mathrm{e}-4$ & $8.60 \mathrm{e}-4$ & 1.1384 \\
0.8 & $1.12 \mathrm{e}-5$ & $2.26 \mathrm{e}-5$ & $4.72 \mathrm{e}-5$ & $1.13 \mathrm{e}-4$ & $3.19 \mathrm{e}-4$ & 1.2082 \\
0.9 & $1.40 \mathrm{e}-5$ & $2.97 \mathrm{e}-5$ & $6.57 \mathrm{e}-5$ & $1.64 \mathrm{e}-4$ & $4.61 \mathrm{e}-4$ & 1.2591 \\
\hline
\end{tabular}

Table 6: Time convergence rates with the different $\alpha \in(0,1)$ for $(13)$ with exact $V^{1}$ and $V^{2}$ by using the reference solution in Example 4.2

Example 4.3. In this example, we consider the same problem as in Example 4.2. Since the exact solution is available in this example, we shall calculate the experimentally determined convergence rates by using the exact solution instead of using the reference solution as in Example 4.2.

To see the convergence rate without using the reference solution, we have to choose sufficiently small space step size otherwise the spacial error will dominates the total error. In our numerical simulation below, we choose the space step size $h=1 / 2000$ and the same time step size as above. The space is also discretized by using the linear finite element method. In Table 7 , we observe the experimentally determined convergence rate $O(k)$ for the scheme $(7)$.

In Table 8, we consider the modified L1 scheme (8)-(10) and choose space step size $h=1 / 2000$ and the time step size as above. We observe that the convergence rates are indeed almost $O\left(k^{2-\alpha}\right)$ as we expected.

In Table 9, we obtained the experimentally determined convergence rate for the scheme (11)-(14). We again choose the space step size $h=1 / 2000$ and the same time step size as above. We find that the convergence rates are lower than $\left.O\left(k^{3-\alpha}\right)\right)$ for $\alpha<1 / 2$ and almost $\left.O\left(k^{3-\alpha}\right)\right)$ for $\alpha>1 / 2$. We believe 


\begin{tabular}{ccccccc}
$\alpha$ & $k=2^{-8}$ & $k=2^{-7}$ & $k=2^{-6}$ & $k=2^{-5}$ & $k=2^{-4}$ & Rate \\
\hline 0.1 & $3.46 \mathrm{e}-5$ & $6.93 \mathrm{e}-5$ & $1.39 \mathrm{e}-4$ & $2.79 \mathrm{e}-4$ & $5.60 \mathrm{e}-4$ & 1.0043 \\
0.3 & $1.07 \mathrm{e}-4$ & $2.14 \mathrm{e}-4$ & $4.28 \mathrm{e}-4$ & $8.58 \mathrm{e}-4$ & $1.72 \mathrm{e}-3$ & 1.0036 \\
0.8 & $3.53 \mathrm{e}-4$ & $7.07 \mathrm{e}-4$ & $1.42 \mathrm{e}-3$ & $2.84 \mathrm{e}-3$ & $5.74 \mathrm{e}-3$ & 1.0053 \\
0.9 & $4.26 \mathrm{e}-4$ & $8.54 \mathrm{e}-4$ & $1.71 \mathrm{e}-3$ & $3.45 \mathrm{e}-3$ & $7.03 \mathrm{e}-3$ & 1.0111 \\
\hline
\end{tabular}

Table 7: Time convergence rates with the different $\alpha \in(0,1)$ for the L1 scheme $(7)$ by using the exact solution in Example 4.3

\begin{tabular}{ccccccc}
$\alpha$ & $k=2^{-8}$ & $k=2^{-7}$ & $k=2^{-6}$ & $k=2^{-5}$ & $k=2^{-4}$ & Rate \\
\hline 0.1 & $2.10 \mathrm{e}-7$ & $4.20 \mathrm{e}-7$ & $1.32 \mathrm{e}-6$ & $5.17 \mathrm{e}-6$ & $2.21 \mathrm{e}-5$ & 1.6778 \\
0.3 & $1.60 \mathrm{e}-7$ & $5.70 \mathrm{e}-7$ & $1.04 \mathrm{e}-6$ & $1.12 \mathrm{e}-6$ & $2.51 \mathrm{e}-5$ & 1.8299 \\
0.8 & $4.11 \mathrm{e}-5$ & $9.21 \mathrm{e}-5$ & $2.01 \mathrm{e}-4$ & $4.19 \mathrm{e}-4$ & $7.79 \mathrm{e}-4$ & 1.0608 \\
0.9 & $5.22 \mathrm{e}-5$ & $1.09 \mathrm{e}-4$ & $2.18 \mathrm{e}-4$ & $4.11 \mathrm{e}-4$ & $6.47 \mathrm{e}-4$ & 0.9083 \\
\hline
\end{tabular}

Table 8: Time convergence rates with the different $\alpha \in(0,1)$ for the modified L1 scheme (8)-(10) by using the exact solution in Example 4.3

the reason is that the spatial error still dominated the total error for the case $\alpha<1 / 2$. In other words, $h=1 / 2000$ is sufficient to observe the time convergence rate $O\left(k^{2-\alpha}\right)$ for the modified L1 scheme (8)-(10), however to observe the convergence rate $O\left(k^{3-\alpha}\right)$ for the scheme (11)-(14), one may need to choose even smaller space step size $h<1 / 2000$. We shall not produce the numerical experiments for such smaller space step size because of the computational times.

\begin{tabular}{ccccccc}
$\alpha$ & $k=2^{-8}$ & $k=2^{-7}$ & $k=2^{-6}$ & $k=2^{-5}$ & $k=2^{-4}$ & Rate \\
\hline 0.1 & $6.40 \mathrm{e}-8$ & $5.78 \mathrm{e}-8$ & $7.32 \mathrm{e}-9$ & $4.13 \mathrm{e}-7$ & $4.12 \mathrm{e}-6$ & 1.5030 \\
0.3 & $1.48 \mathrm{e}-7$ & $1.35 \mathrm{e}-7$ & $2.03 \mathrm{e}-8$ & $9.83 \mathrm{e}-7$ & $1.02 \mathrm{e}-5$ & 1.5260 \\
0.8 & $1.54 \mathrm{e}-7$ & $1.49 \mathrm{e}-8$ & $8.22 \mathrm{e}-7$ & $6.40 \mathrm{e}-6$ & $4.85 \mathrm{e}-5$ & 2.0739 \\
0.9 & $1.44 \mathrm{e}-7$ & $1.31 \mathrm{e}-6$ & $6.61 \mathrm{e}-6$ & $3.18 \mathrm{e}-5$ & $1.64 \mathrm{e}-4$ & 2.5371 \\
\hline
\end{tabular}

Table 9: Time convergence rates with the different $\alpha \in(0,1)$ for $(11)-(14)$ by using the exact solution in Example 4.3

\section{Acknowledgements}

The authors are grateful to the Reviewers and the Associate Editor for their helpful comments. The second author thanks Prof. Neville J. Ford for his consistent support and encouragements for this research.

\section{References}

[1] E. E. Adams and L. W. Gelhar, Field study of dispersion in a heterogeneous aquifer: 2. Spatial moments analysis, Water Res. Research, 28 (1992), pp. 3293-3307.

[2] E. Bazhlekova, B. Jin, R. Lazarov and Z. Zhou, An analysis of the Rayleigh-Stokes problem for a generalized second-grade fluid, Numer. Math., 131 (2015), pp. 1-31.

[3] F. Chen, Q. Xu, and J. S. Hesthaven, A multi-domain spectral method for time-fractional differential equations, J. Comput. Phys., 293(2015), pp. 157-172. 
[4] S. Chen, J. Shen, and L.-L. Wang, Generalized Jacobi functions and their applications to fractional differential equations, Math. Comp., 85(2016), pp. 1603-1638.

[5] X. Chen, F. Zeng, and G.E.Karniadakis, A tunable finite difference method for fractional differential equations with non-smooth solutions, Comput. Methods Appl. Mech. Engrg., 318(2017), pp. 193-214.

[6] W. Deng and J. S. Hesthaven, Local discontinuous Galerkin methods for fractional ordinary differential equations, BIT, 55(2015), pp. 967-985.

[7] K. Diethelm, An algorithm for the numerical solution of differential equations of fractional order, Electronic Transactions on Numerical Analysis, 5(1997), pp. 1-6.

[8] K. Diethelm, Generalized compound quadrature formulae for finite-part integral, IMA J. Numer. Anal., 17 (1997) 479493.

[9] P. Flajolet, Singularity analysis and asymptotics of Bernoulli sums, Theoret. Comput,Sci,,215(1999), pp. 371-381.

[10] N. J. Ford and K. Pal and Y. Yan, An algorithm for the numerical solution of space-fractional partial differential equations, Computational Methods in Applied Mathematics, 15(2015), pp. 497-514.

[11] N. J. Ford, J. Xiao and Y. Yan, A finite element method for time-fractional partial differential equations, Fract. Calc. Appl. Anal., 14(2011), pp. 454-474.

[12] G.-H. Gao, Z.-Z. Sun and H.- W. Zhang, A new fractional numerical differentiation formula to approximate the Caputo fractional derivative and its applications, J. Comput. Phys., 259 (2014), 33-50.

[13] R. Gorenflo and F. Mainardi, Random walk models for space fractional diffusion processes, Fract. Calc. Appl. Anal., 1(1998), pp. 167-191.

[14] Y. Hatano and N. Hatano, Dispersive transport of ions in column experiments: An explanation of long-tailed profiles, Water Res. Research, 34(1998), pp. 1027-1033.

[15] B. Jin, R. Lazarov and Z. Zhou, Error estimates for a semidiscrete finite element method for fractional order parabolic equations, SIAM J. Numer. Anal., 51(2013), pp. 445-466.

[16] B. Jin, R. Lazarov and Z. Zhou, An analysis of the L1 scheme for the subdiffusion equation with nonsmooth data, IMA J. of Numer. Anal., 36(2016), pp. 197-221.

[17] B. Jin, B. Li, and Z. Zhou, An analysis of the Crank-Nicolson method for subdiffusion, IMA J. of Numer. Anal., in press, 2017, arXiv:1607.06948.

[18] B. Jin, B. Li, and Z. Zhou, Correction of high-order BDF convolution quadrature for fractional evolution equations, preprint, arXiv:1703.08808, 2017.

[19] T. A. M. Langlands and B. I. Henry, The accuracy and stability of an implicit solution method for the fractional diffusion equation, J. Comput. Phys., 205(2005), pp. 719-736.

[20] C. Li and H. Ding, Higher order finite difference method for the reaction and anomalous-diffusion equation, Appl. Math. Model., 38 (2014), pp. 3802-3821.

[21] Y. Lin and C. Xu, Finite difference/spectral approximations for the time-fractional diffusion equation, J. Comput. Phys., 225(2007), pp. 1533-1552.

[22] Lv, C., Xu, C.: Error analysis of a high order method for time-fractional diffusion equations. SIAM J. Sci. Comput. 38, A2699-A2724 (2016)

[23] Ch. Lubich, I.H. Sloan and V. Thomée, Nonsmooth data error estimate for approximations of an evolution equation with a positive-type memory term, Math. Comp., 65(1996), pp. 1-17.

[24] W. McLean and K. Mustapha, Time-stepping error bounds for fractional diffusion problems with non-smooth initial data, J. Comput. Phys., 293(2015), pp. 201-217.

[25] K. Mustapha, Time-stepping discontinuous Galerkin methods for fractional diffusion problems, Numer. Math., 130(2015), pp. $497-516$

[26] K. Mustapha, B. Abdallah, and K. M. Furati, A discontinuous Petrov-Galerkin method for time-fractional diffusion equations, SIAM J. Numer. Anal., 52(2014), pp. 2512-2529.

[27] K. Mustapha and W. McLean, Uniform convergence for a discontinuous Galerkin, time-stepping method applied to a fractional diffusion equation, IMA J. of Numer. Anal., 32(2012), pp. 906-925.

[28] K. Mustapha and D. Schötzau, Well-posedness of hp-version discontinuous Galerkin methods for fractional diffusion wave equations, IMA J. Numer. Anal., 34(2014), pp. 1426-1446.

[29] R. Nigmatulin, The realization of the generalized transfer equation in a medium with fractal geometry, Phys. Stat. Sol. B, 133(1986), pp. 425-430.

[30] I. Podlubny, Fractional Differential Equations, Mathematics in Science and Engineering, Vol. 198, Academic Press, 1999.

[31] J. Quintana-Murillo and S. B. Yuste, A finite difference method with non-uniform timesteps for fractional diffusion and diffusion-wave equations, The European Physical Journal Special Topics, 222 (2013), 1987-1998.

[32] K. Sakamoto and M. Yamamoto, Initial value/boundary value problems for fractional diffusion-wave equations and applications to some inverse problems, J. Math. Anal. Appl., 382(2011), pp. 426-447,

[33] M. Stynes, Too much regularity may force too much uniqueness, Fractional Calculus and Applied Analysis, 19(2016), 1554-1562.

[34] M. Stynes, E. O'riordan and J. L. Gracia, Error analysis of a finite difference method on graded meshes for a timefractional diffusion equation, SIAM J. Numer. Anal., 55(2017), pp. 1057-1079.

[35] Z.-Z. Sun and X. Wu, A fully discrete scheme for a diffusion wave system, Appl. Numer. Math., 56(2011), pp. 193-209.

[36] Y. Yan, M. Khan and N. J. Ford, An analysis of the modified scheme for the time-fractional partial differential equations with nonsmooth data, Submitted for publication, 2016.

[37] Y. Yan, K. Pal and N. J. Ford, Higher order numerical methods for solving fractional differential equations, BIT Numer. Math, 54(2014), pp. 555-584 
[38] S.B. Yuste and L. Acedo, An explicit finite difference method and a new von Neumann-type stability analysis for fractional diffusion equation, SIAM J. Numer. Anal.,42(2005), pp. 1862-1874.

[39] S. B. Yuste and J. Quintana-Murillo, Fast, accurate and robust adaptive finite difference methods for fractional diffusion equations, Numer. Algor., 71 (2016), 207-228.

[40] V. Thomée, Galerkin Finite Element Methods for Parabolic Problems, Springer - Verlag, Berlin, 2007.

[41] M. Zayernouri, M. Ainsworth, and G. E. Karniadakis, A unified Petrov-Galerkin spectral method for fractional pdes, Comput. Methods Appl. Mech. Engrg., 283(2015), pp. 1545-1569.

[42] M. Zayernouri and G. E. Karniadakis, Fractional spectral collocation method, SIAM J. Sci. Comput., 36(2014), pp. A40A62.

[43] F. Zeng, C. Li, F. Liu and I. Turner, The use of finite difference/element approaches for solving the time-fractional subdiffusion equation, SIAM J. Sci. Comput., 35(2013), pp. A2976-A3000.

[44] F. Zeng, Z. Zhang, and G. E. Karniadakis, Second-order numerical methods for multi-term fractional differential equations: Smooth and non-smooth solutions, arXiv: 1701.00996, 2017.

\section{Appendix}

\subsection{Proof of Lemma 2.4}

Note that the Riemann-Liouville fractional derivative ${ }_{0}^{R} D_{t}^{\alpha} u(t), 0<\alpha<1$ may be written as

$$
{ }_{0}^{R} D_{t}^{\alpha} u(t)=\frac{1}{\Gamma(1-\alpha)} \frac{d}{d t} \int_{0}^{t}(t-s)^{-\alpha} u(s) d s=\frac{1}{\Gamma(-\alpha)} \oint_{0}^{t}(t-s)^{-\alpha-1} u(s) d s,
$$

where the integral $\oint$ must be interpreted as a Hadamard finite-part integral, see e.g., [8, (1.6)].

At $t=t_{n}, n=5,6, \ldots, N$, we have

$$
\begin{aligned}
{ }_{0}^{R} D_{t}^{\alpha} u\left(t_{n}\right) & =\frac{1}{\Gamma(-\alpha)} \oint_{0}^{t_{n}}\left(t_{n}-s\right)^{-\alpha-1} u(s) d s=\frac{t_{n}^{-\alpha}}{\Gamma(-\alpha)} \oint_{0}^{1} w^{-\alpha-1} u\left(t_{n}-t_{n} w\right) d w \\
& =\frac{t_{n}^{-\alpha}}{\Gamma(-\alpha)} \sum_{j=1}^{n} \oint_{w_{j-1}}^{w_{j}} w^{-\alpha-1} u\left(t_{n}-t_{n} w\right) d w .
\end{aligned}
$$

Denote $g(w)=u\left(t_{n}-t_{n} w\right)$ and approximate $g(w)$ by the piecewise quadratic interpolation polynomial $g_{2}(w)$ defined on the nodes $w_{l}=\frac{l}{n}, l=0,1,2, \ldots, n$ by

$$
\begin{aligned}
g_{2}(w) & =\frac{\left(w-w_{1}\right)\left(w-w_{2}\right)}{\left(w_{0}-w_{1}\right)\left(w_{0}-w_{2}\right)} g\left(w_{0}\right)+\frac{\left(w-w_{0}\right)\left(w-w_{2}\right)}{\left(w_{1}-w_{0}\right)\left(w_{1}-w_{2}\right)} g\left(w_{1}\right) \\
& +\frac{\left(w-w_{0}\right)\left(w-w_{1}\right)}{\left(w_{2}-w_{0}\right)\left(w_{2}-w_{1}\right)} g\left(w_{2}\right), \quad \text { for } w \in\left[w_{0}, w_{1}\right]
\end{aligned}
$$

and

$$
\begin{aligned}
g_{2}(w) & =\frac{\left(w-w_{j-1}\right)\left(w-w_{j}\right)}{\left(w_{j-2}-w_{j-1}\right)\left(w_{j-2}-w_{j}\right)} g\left(w_{j-2}\right)+\frac{\left(w-w_{j-2}\right)\left(w-w_{j}\right)}{\left(w_{j-1}-w_{j-2}\right)\left(w_{j-1}-w_{j}\right)} g\left(w_{j-1}\right) \\
& +\frac{\left(w-w_{j-2}\right)\left(w-w_{j-1}\right)}{\left(w_{j}-w_{j-2}\right)\left(w_{j}-w_{j-1}\right)} g\left(w_{j}\right), \quad \text { for } w \in\left[w_{j-1}, w_{j}\right], j=2,3, \ldots, n .
\end{aligned}
$$

More precisely, on $\left[w_{j-1}, w_{j}\right], j=2,3, \ldots, n, g(w)$ is approximated by the quadratic interpolation polynomial $g_{2}(w)$ determined by $g\left(w_{j-2}\right), g\left(w_{j-1}\right), g\left(w_{j}\right)$, and on $\left[w_{0}, w_{1}\right], g(w)$ is approximated by the quadratic interpolation polynomial $g_{2}(w)$ determined by $g\left(w_{0}\right), g\left(w_{1}\right), g\left(w_{2}\right)$.

By [8, Theorem 2.4], we have the following error estimates

$$
{ }_{0}^{R} D_{t}^{\alpha} u\left(t_{n}\right)=\frac{t_{n}^{-\alpha}}{\Gamma(-\alpha)} \oint_{0}^{1} w^{-\alpha-1} g(w) d w=\frac{t_{n}^{-\alpha}}{\Gamma(-\alpha)} \oint_{0}^{1} w^{-\alpha-1} g_{2}(w) d w+O\left(k^{3-\alpha}\right) .
$$


We next calculate the integral on the right side of (37). Note that

$$
\oint_{0}^{1} w^{-\alpha-1} g_{2}(w) d w=\oint_{0}^{w_{1}} w^{-\alpha-1} g_{2}(w) d w+\sum_{j=2}^{n} \int_{w_{j-1}}^{w_{j}} w^{-\alpha-1} g_{2}(w) d w
$$

where only the first integral on the right side of (38) is in the sense of the Hadamard finite-part integral and the other integrals on the right side of (38) are in the usual sense. Further we have

$$
\oint_{0}^{w_{1}} w^{-\alpha-1} g_{2}(w) d w=\oint_{0}^{w_{1}} w^{-\alpha-1}\left(g_{2}(0)+\int_{0}^{w_{1}} g_{2}^{\prime}(y) d y\right) d w .
$$

Note that, by using the definition of Hadamard finite-part integral, [8]

$$
\oint_{0}^{w_{1}} w^{-\alpha-1} d w=\frac{w_{1}^{-\alpha}}{-\alpha}
$$

we therefore obtain

$$
\oint_{0}^{w_{1}} w^{-\alpha-1} g_{2}(w) d w=\frac{w_{1}^{-\alpha}}{-\alpha} g_{2}(0)+\int_{0}^{w_{1}} w^{-\alpha-1}\left(\int_{0}^{w_{1}} g_{2}^{\prime}(y) d y\right) d w
$$

where

$$
g_{2}^{\prime}(y)=\left(n^{2} / 2\right)\left(2 y-\left(w_{1}+w_{2}\right)\right) g(0)-n^{2}\left(2 y-w_{2}\right) g\left(w_{1}\right)+\left(n^{2} / 2\right)\left(2 y-w_{1}\right) g\left(w_{2}\right) .
$$

Hence

$$
\oint_{0}^{w_{1}} w^{-\alpha-1} g_{2}(w) d w=\frac{1}{(-\alpha)(-\alpha+1)(-\alpha+2) n^{-\alpha}}\left((2-\alpha / 2) g(0)+(-\alpha)(3-\alpha) g\left(w_{1}\right)+(\alpha / 2) g\left(w_{2}\right)\right) .
$$

For the other integrals in (38), we have, with $j=2,3, \ldots, n$,

$$
\begin{aligned}
\int_{w_{j-1}}^{w_{j}} w^{-\alpha-1} g_{2}(w) d w= & \left(n^{2} / 2\right) g\left(w_{j-2}\right) \int_{w_{j-1}}^{w_{j}} w^{-\alpha-1}\left(w-w_{j-1}\right)\left(w-w_{j}\right) d w \\
& -n^{2} g\left(w_{j-1}\right) \int_{w_{j-1}}^{w_{j}} w^{-\alpha-1}\left(w-w_{j-2}\right)\left(w-w_{j}\right) d w \\
& +\left(n^{2} / 2\right) g\left(w_{j}\right) \int_{w_{j-1}}^{w_{j}} w^{-\alpha-1}\left(w-w_{j-2}\right)\left(w-w_{j-1}\right) d w \\
= & \frac{1}{(-\alpha)(-\alpha+1)(-\alpha+2) n^{-\alpha}}\left(\frac{1}{2} E_{j} g\left(w_{j-2}\right)-F_{j} g\left(w_{j-1}\right)+\frac{1}{2} G_{j} g\left(w_{j}\right)\right),
\end{aligned}
$$

where

$$
\begin{aligned}
& E_{j}=2 j^{-\alpha+2}-(-\alpha+2) j^{-\alpha+1}-2(j-1)^{-\alpha+2}-(-\alpha+2)(j-1)^{-\alpha+1} \\
& F_{j}=2 j^{-\alpha+2}-2(-\alpha+2) j^{-\alpha+1}-2(j-1)^{-\alpha+2}+(-\alpha+1)(-\alpha+2)(j-1)^{-\alpha}, \\
& G_{j}=2 j^{-\alpha+2}-3(-\alpha+2) j^{-\alpha+1}-2(j-1)^{-\alpha+2}+(-\alpha+2)(j-1)^{-\alpha+1}+2(-\alpha+1)(-\alpha+2) j^{-\alpha} .
\end{aligned}
$$

Thus we get

$$
\oint_{0}^{1} w^{-\alpha-1} g_{2}(w) d w=\sum_{j=0}^{n} \alpha_{j n} g\left(w_{j}\right)
$$


where

$$
(-\alpha)(-\alpha+1)(-\alpha+2) n^{-\alpha} \alpha_{j n}=\left\{\begin{array}{lr}
2-\frac{1}{2} \alpha+\frac{1}{2} E_{2}, & \text { for } j=0, \\
(-\alpha)(3-\alpha)+\frac{1}{2} E_{3}-F_{2}, & \text { for } j=1, \\
\frac{1}{2} \alpha+\frac{1}{2} E_{4}-F_{3}+\frac{1}{2} G_{2}, & \text { for } j=2, \\
\frac{1}{2} E_{j+2}-F_{j+1}+\frac{1}{2} G_{j}, & \text { for } j=3, \ldots, n-2, \\
-F_{n}+\frac{1}{2} G_{n-1}, & \text { for } j=n-1, \\
\frac{1}{2} G_{n} . & \text { for } j=n .
\end{array}\right.
$$

Therefore we have

$$
\begin{aligned}
{ }_{0}^{R} D_{t}^{\alpha} u\left(t_{n}\right) & =\frac{t_{n}^{-\alpha}}{\Gamma(-\alpha)} \oint_{0}^{1} w^{-\alpha-1} g_{2}(w) d w+O\left(k^{3-\alpha}\right)=\frac{t_{n}^{-\alpha}}{\Gamma(-\alpha)} \sum_{j=0}^{n} \alpha_{j n} g\left(w_{j}\right)+O\left(k^{3-\alpha}\right) \\
& =k^{-\alpha} \sum_{j=0}^{n} \frac{(-\alpha)(-\alpha+1)(-\alpha+2) n^{-\alpha} \alpha_{j n}}{\Gamma(3-\alpha)} g\left(w_{j}\right)+O\left(k^{3-\alpha}\right) \\
& =k^{-\alpha} \sum_{j=0}^{n} w_{j n} u\left(t_{n-j}\right)+O\left(k^{3-\alpha}\right),
\end{aligned}
$$

where, with $j=0,1,2, \ldots, n, n=5,6, \ldots, N$,

$$
\Gamma(3-\alpha) w_{j, n}=(-\alpha)(-\alpha+1)(-\alpha+2) n^{-\alpha} \alpha_{j n} .
$$

More precisely, for $j=0$, we have

$$
\Gamma(3-\alpha) w_{0, n}=2-\frac{\alpha}{2}+\frac{1}{2} E_{2}=\left(1+\frac{\alpha}{2}\right) 2^{1-\alpha} .
$$

For $j=1$, we have

$$
\Gamma(3-\alpha) w_{1, n}=(-\alpha)(3-\alpha)+\frac{1}{2} E_{3}-F_{2}=\left(2+\frac{\alpha}{2}\right) 3^{1-\alpha}-\left(3+\frac{3}{2} \alpha\right) 2^{1-\alpha} .
$$

For $j=2$, we have

$$
\Gamma(3-\alpha) w_{2, n}=\frac{1}{2} \alpha+\frac{1}{2} E_{4}-F_{3}+\frac{1}{2} G_{2}=\left(3+\frac{\alpha}{2}\right) 4^{1-\alpha}-\left(6+\frac{3}{2} \alpha\right) 3^{1-\alpha}+\left(3+\frac{3}{2} \alpha\right) 2^{1-\alpha} .
$$

For $j=3,4, \ldots, n-2$,

$$
\begin{aligned}
\Gamma(3-\alpha) w_{j, n}= & \frac{1}{2} E_{j+2}-F_{j+1}+\frac{1}{2} G_{j}=(j+2)^{2-\alpha}-\left(1-\frac{\alpha}{2}\right)(j+2)^{1-\alpha}-3(j+1)^{2-\alpha} \\
& +\left(3-\frac{3}{2} \alpha\right)(j+1)^{1-\alpha}+3 j^{2-\alpha}-\left(3-\frac{3 \alpha}{2}\right) j^{1-\alpha}-(j-1)^{2-\alpha}+\left(1-\frac{\alpha}{2}\right)(j-1)^{1-\alpha} .
\end{aligned}
$$

For $j=n-1$, we have

$$
\Gamma(3-\alpha) w_{j, n}=(4-2 n-2 \alpha) n^{1-\alpha}+\left(3 n-6+\frac{3 \alpha}{2}\right)(n-1)^{1-\alpha}-\left(n+\frac{\alpha}{2}-3\right)(n-2)^{1-\alpha},
$$

For $j=n$, we have

$$
\Gamma(3-\alpha) w_{n, n}=\left(n-3+\frac{3 \alpha}{2}\right) n^{1-\alpha}+(1-\alpha)(2-\alpha) n^{-\alpha}-\left(n-2+\frac{\alpha}{2}\right)(n-1)^{1-\alpha} .
$$

Together these estimates complete the proof of Lemma 2.4 . 


\subsection{Proof of Lemma 2.8}

We first show (18). It is sufficient to show

$$
\left|\mu\left(e^{-w}\right)-1\right| \leq C|w|^{3-\alpha}, \quad \text { as } w \rightarrow 0 .
$$

Note that, by Lemma 2.5,

$$
\begin{aligned}
\mu\left(e^{-w}\right)-1 & =\left(\frac{e^{-w}}{1-e^{-w}}+a_{1} e^{-w}+a_{2} e^{-2 w}\right)\left(\sum_{j=0}^{\infty} w_{j}\left(e^{-w}\right)^{j}\right)^{\frac{1}{\alpha}}-1 \\
& =\left(\frac{e^{-w}}{1-e^{-w}}+a_{1} e^{-w}+a_{2} e^{-2 w}\right)\left(w^{\alpha}+d_{2} w^{3}+d_{3} w^{3+\alpha}+\ldots\right)^{\frac{1}{\alpha}}-1 \\
& =\left(-1 / 2+a_{1}+a_{2}\right) w+\left(1 / 12-a_{1}-2 a_{2}\right) w^{2}+C w^{3-\alpha}+C_{1} w^{3}+\ldots
\end{aligned}
$$

Since $a_{1}=11 / 12, a_{2}=-5 / 12$, we have

$$
\lim _{w \rightarrow 0} \frac{\mu\left(e^{-w}\right)-1}{w^{3-\alpha}}=C
$$

which implies (18).

Next we show (19). Note that

$$
\frac{|z|}{\left|z_{k}\right|}=\frac{|z|}{\left|\frac{\delta\left(e^{-z k}\right)}{k}\right|}=\frac{|z k|}{\left|\delta\left(e^{-z k}\right)\right|} .
$$

To show (19), it suffices to prove $\frac{|z k|}{\left|\delta\left(e^{-z k}\right)\right|}$ has limit as $|z k| \rightarrow 0$, which follows from

$$
\begin{aligned}
\lim _{w \rightarrow 0} \frac{w}{\delta\left(e^{-w}\right)} & =\lim _{w \rightarrow 0} \frac{w}{\left(\sum_{j=0}^{\infty} w_{j}\left(e^{-w}\right)^{j}\right)^{\frac{1}{\alpha}}}=\lim _{w \rightarrow 0} \frac{w}{\left(w^{\alpha}+d_{2} w^{3}+d_{3} w^{3+\alpha}+\ldots\right)^{\frac{1}{\alpha}}} \\
& =\lim _{w \rightarrow 0} \frac{1}{\left(1+d_{2} w^{3-\alpha}+\ldots\right)^{\frac{1}{\alpha}}}=1 .
\end{aligned}
$$

Hence we have proved, for any fixed constant $M>0$, there exists a constant $C$ such that

$$
\frac{|z|}{\left|z_{k}\right|} \leq C, \forall|z k| \leq M
$$

Similarly we may show $\frac{\left|z_{k}\right|}{|z|} \leq C, \forall|z k| \leq M$. Thus we get (19).

We now show (20). Note that,

$$
\begin{aligned}
z_{k}-z & =\frac{\delta\left(e^{-z k}\right)}{k}-z=\frac{\delta\left(e^{-z k}\right)-z k}{k}=\frac{\left(\sum_{j=0}^{\infty} w_{j}\left(e^{-z k}\right)^{j}\right)^{\frac{1}{\alpha}}-z k}{k} \\
& =\frac{(z k)\left(1+d_{2}(z k)^{3-\alpha}+\ldots\right)^{\frac{1}{\alpha}}-z k}{k}=\frac{(z k)\left(1+\frac{d_{2}}{\alpha}(z k)^{3-\alpha}+\ldots\right)-z k}{k} \\
& =O\left(k^{3-\alpha} z^{4-\alpha}\right)
\end{aligned}
$$

Thus we have, following the proof in $[23,(4.6)]$ and noting $\left\|K^{\prime}(z)\right\| \leq C|z|^{-2}$ in $[23,(3.12)]$,

$$
\left\|K\left(z_{k}\right)-K(z)\right\| \leq C|z|^{-2} k^{3-\alpha}|z|^{4-\alpha} \leq C k^{3-\alpha}|z|^{2-\alpha} .
$$

Finally we show (21). Following the same proof as in [36, Lemma 3.12], we have, noting that $\left|K\left(z_{k}\right)\right| \leq$ $C\left|z_{k}\right|^{-1} \leq C|z|^{-1}$

$$
\begin{aligned}
\left\|\mu(\zeta) K\left(z_{k}\right)-K(z)\right\| & \leq\left\|(\mu(\zeta)-1) K\left(z_{k}\right)\right\|+\left\|K\left(z_{k}\right)-K(z)\right\| \\
& \leq|z k|^{3-\alpha} C|z|^{-1}+C k^{3-\alpha}|z|^{2-\alpha} \leq C k^{3-\alpha}|z|^{2-\alpha}
\end{aligned}
$$

Together these estimates complete the proof of Lemma 2.8 . 